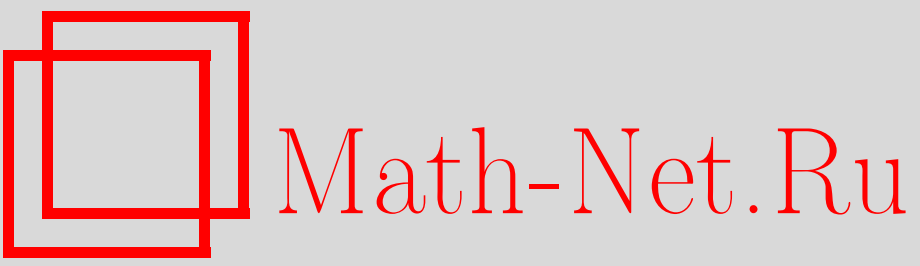

В. А. Солонников, Об оценках решений нестационарной задачи Стокса в анизотропных пространствах С. Л. Соболева и об оценках резольвенты оператора Стокса, УМН, 2003, том 58, выпуск 2, 123-156

DOI: https://doi.org/10.4213/rm613

Использование Общероссийского математического портала Math-Net.Ru подразумевает, что вы прочитали и согласны с пользовательским соглашением

http://www.mathnet.ru/rus/agreement

Параметры загрузки:

IP : 34.227 .88 .159

26 апреля 2023 г., $17: 01: 48$ 


\section{ОБ ОЦЕНКАХ РЕШЕНИЙ НЕСТАЦИОНАРНОЙ ЗАДАЧИ СТОКСА В АНИЗОТРОПНЫХ ПРОСТРАНСТВАХ С. Л. СОБОЛЕВА И ОБ ОЦЕНКАХ РЕЗОЛЬВЕНТЫ ОПЕРАТОРА СТОКСА}

\section{В. А. Солонников}

В работе, которая в значительной степени носит обзорный характер, приведено доказательство коэрцитивной оценки в пространствах Соболева со смешанной нормой для решения нестационарной задачи Стокса (с ненулевой дивергенцией) в ограниченных и внешних областях, а также вытекающей из нее оценки резольвенты оператора Стокса. В доказательствеиспольуется явное представление решения задачи в полупространстве через матрицу Грина, для элементов которой вьводятся поточечные оценки.

Библиография: 26 названий.

\section{СОДЕРЖАНИЕ}

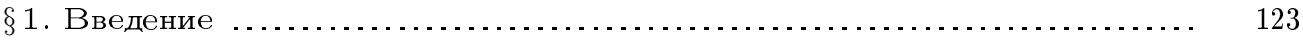

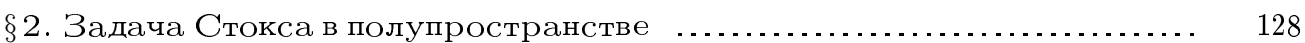

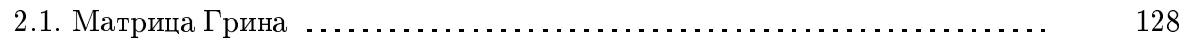

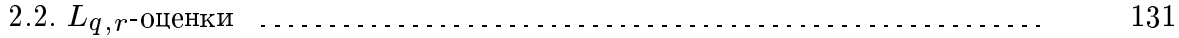

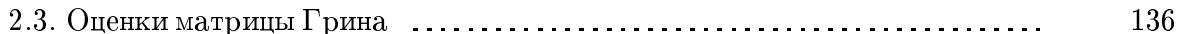

§3. Доказательство теорем $1.1,1.2$............................... 140

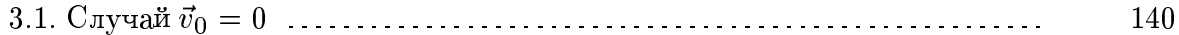

3.2. Задача для системы Стокса с ненулевой дивергенцией $\quad . . . . . . . . . . . . \quad 145$

$\S 4$. Оценка резольвенты оператора Стокса ........................... 151

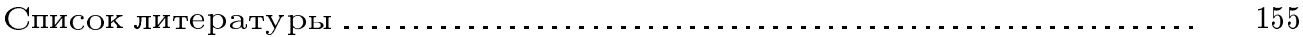

\section{$\S$ 1. Введение}

Настоящая работа посвящена нестационарной задаче Стокса

$$
\begin{gathered}
\vec{v}_{t}-\nu \Delta \vec{v}+\nabla p=\vec{f}, \quad \operatorname{div} \vec{v}=0, \quad x \in \Omega, \quad t \in[0, T), \\
\left.\vec{v}\right|_{t=0}=\vec{v}_{0}(x),\left.\quad \vec{v}\right|_{x \in S}=0
\end{gathered}
$$

в ограниченной или внешней области $\Omega_{\mathbb{R}}^{n}, n \geqslant 2$, с $C^{2}$-гладкой компактной гранищей $S$. Задача (1.1) часто записывается в виде операторного уравнения

$$
v_{t}+A v=f,\left.\quad v\right|_{t=0}=v_{0}
$$


в подходяшем банаховом пространстве соленоидальных векторных полей. Здесь $A=$ $-\nu Q \Delta, Q-$ проектор на подпространство векторных полей, удовлетворяюших (чаще всего в слабом смысле) условиям

$$
\operatorname{div} \vec{f}=0,\left.\quad \vec{f} \cdot \vec{n}\right|_{S}=0,
$$

где $\vec{n}(x)$ - единичная нормаль к $S$. Оператор $A$ назьвается оператором Стокса.

В работе излагаются оценки решения задачи (1.1) в пространствах Соболева $W_{q, r}^{2,1}\left(Q^{T}\right), Q^{T}=\Omega \times(0, T], q, r>1$, и их приложение к оценкам решения стационарной задачи

$$
\begin{gathered}
\lambda \vec{v}-\nu \Delta \vec{v}+\nabla p=\vec{f}(x), \quad \operatorname{div} \vec{v}=0, \quad x \in \Omega, \\
\left.\vec{v}\right|_{S}=0
\end{gathered}
$$

(т.е. уравнения $\lambda v+A v=f$ ), которые позволяют заключить, что оператор Стокса порождает аналитическую полугруппу в $L_{q}(\Omega)$. В случае $q=r$ такие оценки были получены в $60-\mathrm{x}-70$-х годах в лаборатории математической физики ЛОМИ, возглавляемой профессором О. А. Ладыженской (см. [1]-[4]). Они нашли свое обобшение, усиление и применение в многочисленных последуюших работах, выполняемых вплоть до настоящего времени (см., например, [5]-[15]).

Напомним, что под $W_{q, r}^{2,1}\left(Q^{T}\right)$ понимается пространство

$$
L_{r}\left(0, T ; W_{q}^{2}(\Omega)\right) \cap W_{r}^{1}\left(0, T ; L_{q}(\Omega)\right)
$$

с нормой

$$
\begin{gathered}
\|u\|_{W_{q, r}^{2,1}\left(Q^{T}\right)}=\left(\int_{0}^{T}\|u\|_{W_{q}^{2}(\Omega)}^{r} d t\right)^{1 / r}+\left\|u_{t}\right\|_{L_{q, r}\left(Q^{T}\right)}, \\
\|u\|_{L_{q, r}\left(Q^{T}\right)}=\left(\int_{0}^{T}\|u\|_{L_{q}(\Omega)}^{r} d t\right)^{1 / r}=\left(\int_{0}^{T} d t\left(\int_{\Omega}|u(x)|^{q} d x\right)^{r / q}\right)^{1 / r} .
\end{gathered}
$$

Для главной части нормы будем использовать обозначение

$$
\|u\|_{\dot{W}_{q, r}^{2,1}\left(Q^{T}\right)}=\left\|u_{t}\right\|_{L_{q, r}\left(Q^{T}\right)}+\sum_{|j|=2}\left\|D_{x}^{j} u\right\|_{L_{q, r}\left(Q^{T}\right)} .
$$

Пространства векторных полей будем обозначать теми же символами, что и пространства функций.

Известно (см., например, [16]), что если $u \in W_{q, r}^{2,1}\left(Q^{T}\right)$, то при любом $t_{0} \in[0, T]$ функция $u\left(\cdot, t_{0}\right)$ принадлежит пространству О.В. Бесова $B_{q, r}^{2-2 / r}(\Omega)$. При любых $l \in(0,2)$ норму в $B_{q, r}^{l}(\Omega)$ можно определить равенством

$$
\begin{gathered}
\|u\|_{B_{q, r}^{l}(\Omega)}^{l}=\|u\|_{\dot{B}_{q, r}^{l}(\Omega)}+\|u\|_{L_{q}(\Omega)}, \\
\|u\|_{\dot{B}_{q, r}^{l}(\Omega)}^{r}=\int_{|z|<1}|z|^{-n-r l} d z\left(\int_{\Omega(z)}|u(x+2 z)-2 u(x+z)+u(x)|^{q} d x\right)^{r / q},
\end{gathered}
$$

где $\Omega(z)=\{x \in \Omega: x+t z \in \Omega, t \in[0,1]\}$. Имеется много других эквивалентных определений этой нормы. 
Будем говорить, что функция $\varphi \in B_{q, r}^{2-2 / r}(\Omega)$ согласована с нулем на границе, если существует такая функция $u \in W_{q, r}^{2,1}\left(Q^{T}\right)$, что $\left.u\right|_{t=0}=\varphi,\left.u\right|_{x \in S}=0$ и

$$
\|u\|_{W_{q, r}^{2,1}\left(Q_{T}\right)} \leqslant c\|\varphi\|_{B_{q, r}^{2-2 / r}(\Omega)} .
$$

При $2-2 / r>1 / q$ это эквивалентно тому, что $\left.\varphi\right|_{x \in S}=0$, а при $2-2 / r=1 / q$ множество функций, согласованных с нулем, - это множество с конечной нормой

$$
\left\|\varphi^{(0)}\right\|_{B_{q, r}^{2-2 / r}\left(\mathbb{R}^{n}\right)},
$$

где $\varphi^{(0)}(x)=\varphi(x)$ при $x \in \Omega, \varphi^{(0)}(x)=0$ при $x \in \mathbb{R}^{n} \backslash \Omega$. При $2-2 / r<1 / q$ все функции $\varphi \in B_{q, r}^{2-2 / r}(\Omega)$ согласованы с нулем на границе.

Положим

$$
\begin{array}{ll}
\|\varphi\|_{q, r}^{(2-2 / r)}=\|\varphi\|_{B_{q, r}^{2-2 / r}(\Omega)}, & \text { если } 2-2 / r \neq 1 / q, \\
\|\varphi\|_{q, r}^{(2-2 / r)}=\left\|\varphi^{(0)}\right\|_{B_{q, r}^{2-2 / r}\left(\mathbb{R}^{n}\right)}, & \text { если } 2-2 / r=1 / q .
\end{array}
$$

В работе приведено доказательство следуюшей теоремы.

Теорема 1.1. Предположим, что область $\Omega$ ограничена, $\vec{f} \in L_{q, r}\left(Q^{T}\right), \vec{v}_{0} \in$ $B_{q, r}^{2-2 / r}(\Omega)$, вектор $\vec{v}_{0}$ согласован с нулем на граниче и удовлетворяет условиям $\nabla \cdot \vec{v}_{0}=0,\left.\vec{v}_{0} \cdot \vec{n}\right|_{S}=0($ при $2-2 / r<1 / q-$ в слабом смиссле $)$. Тогда задача (1.1) имеет единственное решение $\vec{v} \in W_{q, r}^{2,1}\left(Q^{T}\right), \nabla p \in L_{q, r}\left(Q^{T}\right)$, и выполняется неравенство

$$
\|u\|_{W_{q, r}^{2,1}\left(Q^{T}\right)}+\|\nabla p\|_{L_{q, r}\left(Q^{T}\right)} \leqslant c\left(\|\vec{f}\|_{L_{q, r}\left(Q^{T}\right)}+\left\|\vec{v}_{0}\right\|_{q, \Omega}^{(2-2 / r)}\right) .
$$

Постоянная с от Т не зависит.

В случае внешней области $\Omega$ мы получаем решение в классе функций, которые не обязаны быть элементами $L_{q, r}\left(Q^{T}\right)$ во всей области $Q^{T}$. Мы вводим следующие пространства:

$\widetilde{W}_{q}^{k}(\Omega), k=1,2, \ldots$ : пространство функций $u(x), x \in \Omega$, с конечной нормой

$$
\begin{gathered}
\|u\|_{\widetilde{W}_{q}^{k}(\Omega)}=\|u\|_{\dot{W}_{q}^{k}(\Omega)}+\|u\|_{L_{q}\left(\Omega_{1}\right)}, \\
\|u\|_{\dot{W}_{q}^{k}(\Omega)}^{q}=\sum_{|j|=k}\left\|D^{j} u\right\|_{L_{q}(\Omega)},
\end{gathered}
$$

$\widetilde{W}_{q, r}^{2,1}\left(Q^{T}\right):$ пространство функций $u(x, t),(x, t) \in Q^{T}$, с нормой

$$
\|u\|_{\widetilde{W}_{q, r}^{2,1}\left(Q^{T}\right)}=\|u\|_{\dot{W}_{q, r}^{2,1}\left(Q^{T}\right)}+\|u\|_{L_{q, r}\left(\Omega_{1} \times(0, T)\right)} .
$$

Здесь $\Omega_{1}$ - ограниченная подобласть $\Omega$ такая, что $\left(\Omega \backslash \bar{\Omega}_{1}\right) \cap S=\varnothing$, например, $\Omega_{1}=$ $\left\{x \in \Omega:\left|x-x_{0}\right|<R\right\}$, где $x_{0} \in \mathbb{R}^{n} \backslash \bar{\Omega}$. При изменении $\Omega_{1}$ пространства $\widetilde{W}_{q}^{k}(\Omega)$ и $\widetilde{W}_{q, r}^{2,1}\left(Q^{T}\right)$ не изменяются, а нормы $(1.6),(1.7)$ переходят в эквивалентные. 
Определим теперь пространство следов функций $u \in \widetilde{W}_{q, r}^{2,1}\left(Q^{T}\right)$ на плоскости $t=$ $t_{0} \in[0, T]$. Воспользуемся конечным разбиением единицы в $\Omega$. Пусть $\zeta_{0}, \zeta_{1}, \ldots, \zeta_{M}-$ гладкие функции, заданные в $\mathbb{R}^{n}$ и обладающие следующими свойствами.

1) $\operatorname{supp} \zeta_{k} \subset \omega_{k}=\left\{\left|x-x_{k}\right|<d\right\}, x_{k} \in S, k=1, \ldots, M$,

2) $\sum_{j=1}^{M} \zeta_{j}(x)=1$ при $x \in \Omega_{d / 2}=\{x \in \Omega: \operatorname{dist}(x, S)<d / 2\}$.

Тогда $\zeta_{0}(x)=1-\sum_{j=1}^{M} \zeta_{j}(x)=0$ при $x \in \Omega_{d / 2}$.

3) Каждое $\omega_{k}, k=1, \ldots, M$, может быть отображено в подмножество полупространства $\left(\vec{x}-\vec{x}_{k}\right) \cdot \vec{n}\left(x_{k}\right)>0$ с помошью гладкой замены переменных $z=T_{k} x$; при этом поверхность $\partial \omega_{k} \cap S$ переходит в подмножество касательной плоскости $R_{k}$ : $\left(\vec{x}-\vec{x}_{k}\right) \cdot \vec{n}\left(x_{k}\right)=0$, а точка $x_{k}$ остается инвариантной.

Пусть $\zeta_{j} \varphi \equiv \varphi_{j}, j=0,1, \ldots, M$, и $\widetilde{\varphi}_{j}(z)=\varphi_{j}\left(T_{j} x\right), \varphi_{j}^{(-)}(z)$ - нечетное относительно касательной плоскости продолжение $\widetilde{\varphi}_{j}(z)$ во все пространство $\mathbb{R}^{n}$. Обозначим через $\widetilde{B}_{q, r}^{2-2 / r}(\Omega)$ пространство с нормой

$$
\|\varphi\|_{\widetilde{B}_{q, r}^{2-2 / r}(\Omega)}=\sum_{k=1}^{M}\left\|\varphi_{k}^{(-)}\right\|_{\dot{B}_{q, r}^{2-2 / r}\left(\mathbb{R}^{n}\right)}+\left\|\varphi_{0}\right\|_{\dot{B}_{q, r}^{2-2 / r}\left(\mathbb{R}^{n}\right)}+\|\varphi\|_{L_{q}\left(\Omega_{1}\right)}
$$

где

$$
\|\psi\|_{\dot{B}_{q, r}^{2-2 / r}\left(\mathbb{R}^{n}\right)}^{r}=\int_{\mathbb{R}^{n}} \frac{d z}{|z|^{n+2 r-2}}\left(\int|\psi(x+z)-2 \psi(x)+\psi(x-z)|^{q} d z\right)^{r / q} .
$$

Это пространство состоит из функций, согласованных с нулем на $S$, что вытекает из конечности норм функций $\varphi_{j}^{(-)}$в (1.8). Можно показать, что норма (1.8) эквивалентна норме, использованной в $\left[10 ;(1.3),\left(1.3^{\prime}\right)\right]$. Заменив $\Omega_{1}$ на $\Omega$ в $(1.8)$, получим норму, эквивалентную норме $B_{q, r}^{2-2 / r}(\Omega)$. Для внешних областей доказывается следуюшая теорема.

TEорема 1.2. Пусть $\Omega$ - внешняя область, $\vec{f} \in L_{q, r}\left(Q^{T}\right), \vec{v}_{0} \in \widetilde{B}_{q, r}^{2-2 / r}(\Omega)$, $\nabla \cdot \vec{v}_{0}=0,\left.\vec{v}_{0} \cdot \vec{n}\right|_{S}=0$. Тогда задача (1.1) имеет единственное решение $\vec{v} \in$ $\widetilde{W}_{q, r}^{2,1}\left(Q_{T}\right), \nabla p \in L_{q, r}\left(Q^{T}\right)$, и выполняется неравенство

$$
\|\vec{v}\|_{\widetilde{W}_{q, r}^{2,1}\left(Q^{T}\right)}+\|\nabla p\|_{L_{q, r}\left(Q^{T}\right)} \leqslant c\left(\|\vec{f}\|_{L_{q, r}\left(Q^{T}\right)}+\left\|\vec{v}_{0}\right\|_{\widetilde{B}_{q, r}^{2-2 / r}(\Omega)}\right) .
$$

При $n \geqslant 3, q<n / 2$ постоянная с от $T$ не зависит.

Если $\vec{v}_{0} \in B_{q, r}^{2-2 / r}(\Omega)$, то $\vec{v} \in W_{q, r}^{2,1}\left(Q^{T}\right)$.

В работе [4] была доказана теорема 1.1, а также теорема 1.2 с некоторой зависящей от $T$ постоянной $c$ в неравенстве (1.10) в случае $q=r$. И. Гига и $Г$. Зор [7] доказали оценки (1.5), (1.10) при произвольных $q, r>1$ и показали, что постоянная $c$ в (1.10) может быть выбрана не зависящей от $T$, если $n \geqslant 3, q<n / 2$, а при $q \geqslant n / 2$ получили неравенство

$$
\left\|\vec{v}_{t}\right\|_{L_{q, r}\left(Q^{T}\right)}+\|-\nu \nabla \vec{v}+\nabla p\|_{L_{q, r}\left(Q^{T}\right)} \leqslant c\left(\|\vec{f}\|_{L_{q, r}\left(Q^{T}\right)}+\left\|\vec{v}_{0}\right\|_{\tilde{B}_{q, r}^{2-2 / r}(\Omega)}\right)
$$


также с не зависящей от $T$ постоянной (следует отметить, что пространство начальных данных $\vec{v}_{0}$ определено в [7] иньм образом). Другое доказательство этих результатов было дано в [11]. Как показано в [17], оценка (1.10) с $q=2$ имеет место и при $n=2$. Теоремы 1.1 и 1.2 доказьваются ниже в несколько более общей форме, чем указано вьше, а именно для системы с уравнением $\operatorname{div} \vec{v}=g$ вместо $\operatorname{div} \vec{v}=0$. Задача (1.1) для системы такого вида была рассмотрена в [18].

Переходим к оценкам резольвенты оператора Стокса. В работе [4] было показано, что решение задачи (1.3) подчиняется неравенству

$$
|\lambda|\|\vec{v}\|_{L_{q}(\Omega)}+\|\vec{v}\|_{\dot{W}_{q}^{2}(\Omega)}+\|\nabla p\|_{L_{q}(\Omega)} \leqslant c\|\vec{f}\|_{L_{q}(\Omega)}
$$

с постоянной, не зависящей от $\lambda$, для всех $\lambda \in \Sigma_{\rho, \omega}=\{|\arg \lambda|<\pi / 2+\omega,|\lambda|>\rho\}$ с некоторыми положительными $\omega$ и $\rho$. Это неравенство было вьведено из полученной в той же работе оценки решения нестационарной задачи $(1.1)\left(\mathrm{c} \vec{v}_{0}=0\right)$

$$
\|\vec{v}\|_{W_{q, q}^{2,1}\left(Q^{T}\right)}+\|\nabla p\|_{L_{q}\left(Q^{T}\right)} \leqslant c_{1}\|\vec{f}\|_{L_{q}\left(Q^{T}\right)}+c_{2}\|\vec{v}\|_{L_{q}\left(Q^{T}\right)}
$$

с постоянньми $c_{1}$ и $c_{2}$, не зависяшими от $T$. В случае ограниченной области этот результат, дополненньй несложньм анализом задачи (1.3) при конечных $|\lambda|$, позволяет заключить, что (1.11) выполняется в области $\Sigma_{\rho, 0} \cup\{\mathbb{R} \lambda>-b\}$, где $-b$ - первое собственное число оператора Стокса. В случае внешней области это не имеет места и, ввиду важности оценки резольвенты оператора Стокса для изучения нестационарной задачи, результаты работы [4] подверглись обобщению и усилению. И. Гига [5] получил неравенство

$$
|\lambda|\|\vec{v}\|_{L_{q}(\Omega)} \leqslant c\|\vec{f}\|_{L_{q}(\Omega)}, \quad \lambda \in \Sigma_{\omega, \rho},
$$

для произвольных $\omega, \rho>0$. Это было сделано с помошью сведения задачи (1.3) к задаче для однородной системы с неоднородным краевым условием, которая была изучена методами теории потенциала и теории псевдодифференщиальных операторов. М. Мак-Крекен [19] доказала оценку (1.11) при $\lambda \in \Sigma_{\omega, \rho}, \forall \omega>0$, для случаев $\Omega=\mathbb{R}^{n}$ и $\Omega=\mathbb{R}_{+}^{n}$. Доказательство основано на теореме о мультипликаторах в интегралах Фурье и на представлении решения краевой задачи в $\mathbb{R}_{+}^{n}$ в виде потенциалов. Опираясь на эти результаты, В. Борхерс и Г. Зор [8] доказали оценку (1.13) при $\lambda \in \Sigma_{\omega, 0}$, $\forall \omega>0$, для внешней области. Это позволило, в частности, установить равномерную ограниченность в $L_{q}(\Omega)$ полугрупп, порождаемой оператором Стокса, для любых $t \geqslant 0$ (из результатов работы [4] следовала ограниченность на любом конечном интервале $t \in[0, T])$. Другой метод доказательства этой же оценки при $\lambda \in \Sigma_{\omega, 0}$ с некоторым $\omega>0$, основанньй на изучении нестационарной задачи, содержится в работе [11]. При $n=2$ оценка (1.13) получена методами теории потенциала в [9]. Некоторые результаты работы [8] воспроизводятся ниже в $\S 4$.

Коэрцитивные оценки вида (1.5) широко применяются для изучения нелинейной задачи, они позволили установить тот хорошо известный факт, что слабое решение задачи принадлежит $W_{q, r}^{2,1}\left(Q^{T}\right)$ при $n / q+2 / r=n+1$. Их применение к изучению локальных свойств решения нелинейной задачи можно найти в [14], [15]. 


\section{§. Задача Стокса в полупространстве}

2.1. Матрица Грина. Рассмотрим задачу

$$
\begin{gathered}
\vec{v}_{t}-\nu \Delta \vec{v}+\nabla p=\vec{f}(x, t), \\
\operatorname{div} \vec{v}=0, \quad x \in \mathbb{R}_{+}^{n}=\left\{x_{n}>0\right\}, \quad t \in[0, T), \quad T \leqslant \infty, \\
\left.\vec{v}\right|_{t=0}=0,\left.\quad \vec{v}\right|_{x_{n}=0}=0,
\end{gathered}
$$

считая векторное поле $\vec{f}(x, t)$ гладким и достаточно быстро убывающим при $|x| \rightarrow \infty$. В работе [4] показано (для случая $n=3$, но обший случай рассматривается буквально так же), что если $\vec{f}$ удовлетворяет условиям

$$
\operatorname{div} \vec{f}=0,\left.\quad f_{n}\right|_{x_{n}=0}=0,
$$

то решение задачи (2.1) выражается в виде

$$
\begin{aligned}
& \vec{v}(x, t)=\int_{0}^{t} \int_{\mathbb{R}_{+}^{n}} \mathscr{G}(x, y, t-\tau) \vec{f}(y, \tau) d y d \tau, \\
& p(x, t)=\int_{0}^{t} \int_{\mathbb{R}_{+}^{n}} \overrightarrow{\mathscr{P}}(x, y, t-\tau) \cdot \vec{f}(y, \tau) d y d \tau .
\end{aligned}
$$

Элементы матрищы $\mathscr{G}=\left(G_{i j}\right)_{i, j=1, \ldots, n}$ и компоненты вектора $\overrightarrow{\mathscr{P}}=\left(P_{i}\right)_{i=1, \ldots, n}$ определяются формулами

$$
\begin{aligned}
G_{i j}(x, y ; t)= & \delta_{i j}\left(\Gamma(x-y, t)-\Gamma\left(x-y^{*}, t\right)\right) \\
& +4\left(1-\delta_{j n}\right) \frac{\partial}{\partial x_{j}} \int_{0}^{x_{n}} \int_{\mathbb{R}^{n-1}} \frac{\partial E(x-z)}{\partial x_{i}} \Gamma\left(z-y^{*}, t\right) d z, \\
P_{j}(x, y, t)= & 4 \nu\left(1-\delta_{j n}\right) \frac{\partial}{\partial x_{j}}\left[\int_{\mathbb{R}^{n-1}} \frac{\partial E\left(x^{\prime}-z^{\prime}, x_{n}\right)}{\partial x_{n}} \Gamma\left(z^{\prime}-y^{\prime}, y_{n}, t\right) d z^{\prime}\right. \\
& \left.+\int_{\mathbb{R}^{n-1}} E\left(x^{\prime}-z^{\prime}, x_{n}\right) \frac{\partial \Gamma\left(z^{\prime}-y^{\prime}, y_{n}, t\right)}{\partial y_{n}} d z^{\prime}\right],
\end{aligned}
$$

в которых $x^{\prime}=\left(x_{1}, \ldots, x_{n-1}\right), x^{*}=\left(x^{\prime},-x_{n}\right)$ - точка, симметричная точке $x$ относительно плоскости $x_{n}=0$, a $E(x)$ и $\Gamma(x, t)$ - фундаментальные решения уравнения Лапласа и уравнения теплопроводности соответственно, т.е.

$$
\begin{array}{ll}
E(x)=\frac{1}{2 \pi} \log |x|, & \text { если } n=2, \\
E(x)=-\frac{1}{(n-2)\left|S_{1}\right||x|^{n-2}}, & \text { если } n>2,
\end{array}
$$

$\left|S_{1}\right|=2 \pi^{n / 2} \Gamma(n / 2)$ - плошадь поверхности единичной сферы в $\mathbb{R}^{n}$,

$$
\begin{array}{lr}
\Gamma(x, t)=(4 \pi \nu t)^{-n / 2} \exp \left(-\frac{|x|^{2}}{4 \nu t}\right) & \text { при } t>0, \\
\Gamma(x, t)=0 & \text { при } t<0 .
\end{array}
$$


Если условия (2.2) не вьполнены, то

$$
\begin{gathered}
\vec{v}(x, t)=\int_{0}^{t} \int_{\mathbb{R}_{+}^{n}} \mathscr{G}(x, y, t-\tau) \vec{f}^{\prime}(y, \tau) d y d \tau, \\
p(x, t)=\varphi(x, t)+\int_{0}^{t} \int_{\mathbb{R}_{+}^{n}} \overrightarrow{\mathscr{P}}(x, y, t-\tau) \cdot \vec{f}^{\prime}(y, \tau) d y d \tau,
\end{gathered}
$$

где $\vec{f}^{\prime}$ - проекция $\vec{f}$ на пространство векторных полей, удовлетворяюших (2.2). Известно, что произвольное поле $\vec{f}$ может быть представлено в виде

$$
\begin{gathered}
\vec{f}=\nabla \varphi+\vec{f}^{\prime}, \\
\varphi(x, t)=-\int_{\mathbb{R}_{+}^{n}} \nabla_{y}\left(E(x-y)+E\left(x-y^{*}\right)\right) \cdot \vec{f}(y, t) d y,
\end{gathered}
$$

так что $\vec{f}^{\prime}$ связано с $\vec{f}$ соотношением

$$
\vec{f}^{\prime}(x, t)=\vec{f}(x, t)+\nabla \int_{\mathbb{R}_{+}^{n}} \nabla_{y}\left(E(x-y)+E\left(x-y^{*}\right)\right) \cdot \vec{f}(y, t) d y .
$$

Формулы (2.3), (2.4) были выведены в [4] с помошью преобразования Фурье задачи (2.1) и решения получившейся задачи для системы обыкновенных дифференциальных уравнений в явном виде. Вместо этого мы проверим, что функции $(2.3),(2.4)$ действительно являются решением задачи (2.1), если вьполнены условия (2.2). Запишем компоненты вектора (2.3) в виде

$$
v_{i}(x, t)=u_{i}(x, t)+4 \sum_{j=1}^{n-1} \frac{\partial}{\partial x_{j}} \int_{0}^{x_{n}} \int_{\mathbb{R}^{n-1}} \frac{\partial E(x-z)}{\partial x_{i}} U_{j}^{*}(z, t) d z,
$$

где

$$
U_{j}^{*}(x, t)=\int_{0}^{t} \int_{\mathbb{R}_{+}^{n}} \Gamma\left(x-y^{*}, t-\tau\right) f_{j}(y, \tau) d y d \tau,
$$

a

$$
u_{i}(x, t)=\int_{0}^{t} \int_{\mathbb{R}_{+}^{n}}\left(\Gamma(x-y, t-\tau)-\Gamma\left(x-y^{*}, t-\tau\right)\right) f_{i}(y, \tau) d y d \tau
$$

- решение параболической начально-краевой задачи

$$
\frac{\partial u_{i}}{\partial t}-\nu \Delta u_{i}=f_{i}, \quad x \in \mathbb{R}_{+}^{n},\left.\quad u_{i}\right|_{t=0}=0,\left.\quad u_{i}\right|_{x_{n}=0}=0 .
$$

Ясно, что функции (2.8) удовлетворяют нулевым начальным и краевым условиям.

Далее, из легко доказьваемого соотношения

$$
\sum_{i=1}^{n} \frac{\partial}{\partial x_{i}} \int_{0}^{x_{n}} \int_{\mathbb{R}^{n-1}} \frac{\partial E(x-z)}{\partial x_{i}} h(z) d z=\frac{1}{2} h(x)
$$


следует, что

$$
\begin{aligned}
\operatorname{div} \vec{v} & =\operatorname{div} \vec{u}+2 \sum_{j=1}^{n-1} \frac{\partial U_{j}^{*}}{\partial x_{j}} \\
& =-\int_{0}^{t} \int_{\mathbb{R}_{+}^{n}} \sum_{i=1}^{n} \frac{\partial}{\partial y_{i}}\left(\Gamma(x-y, t-\tau)+\Gamma\left(x-y^{*}, t-\tau\right)\right) f_{i}(y, \tau) d y d \tau=0
\end{aligned}
$$
вследствие условий $(2.2)$. Наконец, вынося производную $\frac{\partial}{\partial x_{i}}$ в $(2.8)$ из-под знака ин-
теграла, получаем

$$
\begin{aligned}
v_{i}(x, t)= & u_{i}(x, t)+4 \sum_{j=1}^{n-1} \frac{\partial^{2}}{\partial x_{i} \partial x_{j}} \int_{0}^{x_{n}} \int_{\mathbb{R}^{n-1}} E(x-z) U_{j}^{*}(z, t) d z \\
& -4 \delta_{i n} \sum_{j=1}^{n-1} \frac{\partial}{\partial x_{j}} \int_{\mathbb{R}^{n-1}} E\left(y^{\prime}, 0\right) U_{j}^{*}\left(x^{\prime}-y^{\prime}, x_{n}, t\right) d y^{\prime}
\end{aligned}
$$

и, следовательно,

$$
\left(\frac{\partial}{\partial t}-\nu \Delta\right) v_{i}=f_{i}-\frac{\partial p}{\partial x_{i}}
$$

где

$$
\begin{aligned}
p(x, t)= & -4 \sum_{j=1}^{n-1} \frac{\partial}{\partial x_{j}}\left(\frac{\partial}{\partial t}-\nu \Delta\right) \int_{0}^{x_{n}} \int_{\mathbb{R}^{n-1}} E(y) U_{j}^{*}(x-y, t) d y \\
= & 4 \nu \sum_{j=1}^{n-1} \frac{\partial^{2}}{\partial x_{j} \partial x_{n}} \int_{\mathbb{R}^{n-1}} E\left(y^{\prime}, x_{n}\right) U_{j}^{*}\left(x^{\prime}-y^{\prime}, 0, t\right) d y^{\prime} \\
& +\left.4 \nu \sum_{j=1}^{n-1} \frac{\partial}{\partial x_{j}} \int_{\mathbb{R}^{n-1}} E\left(y^{\prime}, x_{n}\right) \frac{\partial U_{j}^{*}\left(x^{\prime}-y^{\prime}, r, t\right)}{\partial r}\right|_{r=0} d y^{\prime} \\
= & 4 \nu \sum_{j=1}^{n}\left(\int_{\mathbb{R}^{n-1}} \frac{\partial E\left(x^{\prime}-z^{\prime}, x_{n}\right)}{\partial x_{j}} d z^{\prime} \int_{0}^{t} \int_{\mathbb{R}^{n-1}} \frac{\partial \Gamma\left(z^{\prime}-y^{\prime}, y_{n}, t-\tau\right)}{\partial y_{n}} f_{j}(y, \tau) d y d \tau\right. \\
& \left.+\int_{\mathbb{R}^{n-1}} \frac{\partial E\left(x^{\prime}-z^{\prime}, x_{n}\right)}{\partial x_{n}} d z^{\prime} \int_{0}^{t} \int_{\mathbb{R}^{n-1}} \frac{\partial \Gamma\left(z^{\prime}-y^{\prime}, y_{n}, t-\tau\right)}{\partial z_{j}} f_{j}(y, \tau) d y d \tau\right)
\end{aligned}
$$

что совпадает с (2.4).

При невьполнении условий $(2.2)$ мы представляем $\vec{f}$ в виде $(2.7)$ и присоединяем $\varphi$ к давлению $p$, что приводит к (2.5), (2.6).

Легко также видеть, что решение задачи

$$
\begin{gathered}
\vec{v}_{t}-\nu \Delta \vec{v}+\nabla p=0, \quad \operatorname{div} \vec{v}=0, \quad x \in \mathbb{R}^{n}, \quad t>0, \\
\left.\vec{v}\right|_{t=0}=\vec{v}_{0}(x),\left.\quad \vec{v}\right|_{x_{n}=0}=0
\end{gathered}
$$


с любым гладким убывающим на бесконечности соленоидальным $\vec{v}_{0}$, удовлетворяюшим условию $\left.v_{0 n}\right|_{x_{n}=0}=0$, выражается по формулам

$$
\begin{aligned}
& \vec{v}(x, t)=\int_{\mathbb{R}_{+}^{n}} \mathscr{G}(x, y, t) \vec{v}_{0}(y) d y, \\
& p(x, t)=\int_{\mathbb{R}_{+}^{n}} \overrightarrow{\mathscr{P}}(x, y, t) \cdot \vec{v}_{0}(y) d y .
\end{aligned}
$$

2.2. $L_{q, r}$-оценки. Докажем следуюшее предложение.

ПредЛОЖениЕ 2.1. Решение задачи (2.1), определяемое формулами (2.3), (2.4), подчиняется неравенству

$$
\|\vec{v}\|_{\dot{W}_{q, r}^{2,1}\left(\Pi_{+}^{T}\right)}+\|\nabla p\|_{L_{q, r}\left(\Pi_{T}^{+}\right)} \leqslant c\|\vec{f}\|_{L_{q, r}\left(\Pi_{+}^{T}\right)},
$$

әде $\Pi_{+}^{T}=\mathbb{R}_{+}^{n} \times(0, T), c-$ постоянная, не зависящая от $T, u\|\vec{v}\|_{\dot{W}_{q, r}^{2,1}\left(\Pi_{+}^{T}\right)}-$ главная часть нормы $\|\vec{v}\|_{W_{q, r}^{2,1}\left(\Pi_{+}^{T}\right)}$ :

$$
\|\vec{v}\|_{\dot{W}_{q, r}^{2,1}\left(\Pi_{+}^{T}\right)}=\left\|\vec{v}_{t}\right\|_{L_{q, r}\left(\Pi_{+}^{T}\right)}+\sum_{|j|=2}\left\|D_{x}^{j} \vec{v}\right\|_{L_{q, r}\left(\Pi_{+}^{T}\right)} .
$$

Кроме того,

$$
\|\vec{v}\|_{L_{q, r}\left(\Pi_{+}^{T}\right)} \leqslant c T\|\vec{f}\|_{L_{q, r}\left(\Pi_{+}^{T}\right)} .
$$

ДоКАЗАТЕЛЬСтво. Основную роль в доказательстве неравенства (2.12) играют теорема Кальдерона-Зигмунда и оценка

$$
\|u\|_{\dot{W}_{q, r}^{2,1}\left(\Pi^{T}\right)} \leqslant c\|f\|_{L_{q, r}\left(\Pi^{T}\right)}, \quad \Pi^{T}=\mathbb{R}^{n} \times(0, T),
$$

теплового объемного потенциала

$$
u(x, t)=\int_{0}^{t} \int_{\mathbb{R}^{n}} \Gamma(x-y, t-\tau) f(y, \tau) d y d \tau
$$

(см., например, [20]). Оценим производные $\frac{\partial v_{i}}{\partial t}$ с помошью формулы (2.8), которую запишем в виде

$$
v_{i}(x, t)=u_{i}(x, t)+4 \sum_{j=1}^{n-1} S_{i j} U_{j}^{*}+\frac{2}{n}\left(1-\delta_{i n}\right) U_{i}^{*}(x, t),
$$

где $S_{i j}$ - сингулярный интегральный оператор с разрьвньм ядром

$$
K_{i j}(x)= \begin{cases}\frac{\partial^{2} E(x)}{\partial x_{i} \partial x_{j}}, & \text { если } x_{n}>0, \\ 0, & \text { если } x_{n}<0,\end{cases}
$$


$U_{j}^{*}$ задается формулой $(2.9)$ при $x_{n}>0$ и $U_{j}^{*}=0$ при $x_{n}<0$. Дифференцируя (2.14) по $t$ и применяя (2.13) и теорему Кальдерона-Зигмунда [21], получаем

$$
\left\|\vec{v}_{t}\right\|_{L_{q, r}\left(\Pi_{+}^{T}\right)} \leqslant\left\|\vec{u}_{t}\right\|_{L_{q, r}\left(\Pi_{+}^{T}\right)}+c\left\|\vec{U}_{t}^{*}\right\|_{L_{q, r}\left(\Pi_{+}^{T}\right)} \leqslant c\|\vec{f}\|_{L_{q, r}\left(\Pi_{+}^{T}\right)} .
$$

Рассмотрим теперь $\vec{v}, p$ как решение стационарной задачи

$$
-\nu \Delta \vec{v}+\nabla p=\vec{f}-\vec{v}_{t}, \quad \operatorname{div} \vec{v}=0, \quad x \in \mathbb{R}_{+}^{n},\left.\quad \vec{v}\right|_{x_{n}=0}=0
$$

при любом $t \in(0, T)$. Известная коэрцитивная оценка норм $L_{q}\left(\mathbb{R}_{+}^{n}\right)$ производных $\frac{\partial^{2} \vec{v}}{\partial x_{i} \partial x_{j}}$ и $\nabla p$ и неравенство (2.15) влекут за собой $(2.12)$.

Неравенство $\left(2.12^{\prime}\right)$ легко получается из оценки

$$
\|\vec{v}(\cdot t)\|_{L_{q}\left(\mathbb{R}_{+}^{n}\right)} \leqslant \int_{0}^{t}\left\|\vec{v}_{\tau}(\cdot \tau)\right\|_{L_{q}\left(\mathbb{R}_{+}^{n}\right)} d \tau
$$

откуда следует

$$
\|\vec{v}\|_{L_{q, r}\left(\Pi_{+}^{T}\right)} \leqslant T\left\|\vec{v}_{t}\right\|_{L_{q, r}\left(\Pi_{+}^{T}\right)} \leqslant c T\|\vec{f}\|_{L_{q, r}\left(\Pi_{+}^{T}\right)},
$$

что и требовалось.

Для доказательства (1.5), (1.10) нам нужно рассмотреть более общую задачу

$$
\begin{gathered}
\vec{v}_{t}-\nu \Delta \vec{v}+\nabla p=\vec{f}(x, t), \\
\operatorname{div} \vec{v}=g(x, t), \quad x \in \mathbb{R}_{+}^{n}, \quad t \in[0, T), \\
\left.\vec{v}\right|_{t=0}=\vec{v}_{0}(x),\left.\quad \vec{v}\right|_{x_{n}=0}=0 .
\end{gathered}
$$

Будем считать, что $\vec{f}, g, \vec{v}_{0}$ - гладкие функции, стремяшиеся к нулю при $|x| \rightarrow \infty$ и удовлетворяюшие условиям согласования

$$
\operatorname{div} \vec{v}_{0}(x, 0)=g(x, 0),\left.\quad \vec{v}_{0}\right|_{x_{n}=0}=0 .
$$

Кроме того, предположим, что функция $g(x, t)$ дифференщируема по $t$ и $g_{t} \in L_{r}(0, T$; $\left.\dot{W}_{q}^{-1}\left(\mathbb{R}_{+}^{n}\right)\right)$, т.е.

$$
\left|\int_{\mathbb{R}_{+}^{n}} g_{t}(x, t) \varphi(x) d x\right| \leqslant c\left\|g_{t}(\cdot t)\right\|_{\dot{W}_{q}^{-1}\left(\mathbb{R}_{+}^{n}\right)}\|\nabla \varphi\|_{L_{q^{\prime}}\left(\mathbb{R}_{+}^{n}\right)},
$$

$1 / q^{\prime}=1-1 / q$, и $\left\|g_{t}(\cdot t)\right\|_{\dot{W}_{q}^{-1}\left(\mathbb{R}_{+}^{n}\right)} \in L_{r}(0, T)$. Построим решение задачи $(2.16)$ в виде суммы

$$
\begin{aligned}
& \vec{v}(x, t)=\vec{v}_{1}(x, t)+\vec{v}_{2}(x, t)+\vec{v}_{3}(x, t), \\
& p(x, t)=p_{2}(x, t)+p_{3}(x, t),
\end{aligned}
$$


где

$$
\begin{aligned}
\vec{v}_{1}(x, t) & =\int_{\mathbb{R}_{+}^{n}}\left(\Gamma(x-y, t)-\Gamma\left(x-y^{*}, t\right)\right) \vec{v}_{0}(y) d y \\
& =\int_{\mathbb{R}_{+}^{n}} \Gamma(x-y, t) \vec{v}_{0}^{(-)}(y) d y
\end{aligned}
$$

$\vec{v}_{0}^{(-)}(y)$ - нечетное по $y_{n}$ продолжение $\vec{v}_{0}$ в область $y_{n}<0$, a $\left(\vec{v}_{2}, p_{2}\right)$ и $\left(\vec{v}_{3}, p_{3}\right)$ - решения задач

$$
\begin{gathered}
-\nu \Delta \vec{v}_{2}+\nabla p_{2}=0, \\
\operatorname{div} \vec{v}_{2}=g-\operatorname{div} \vec{v}_{1} \equiv g_{1}(x, t), \quad x \in \mathbb{R}_{+}^{n}, \\
\left.\vec{v}\right|_{x_{3}=0}=0, \\
\vec{v}_{3 t}-\nu \Delta \vec{v}_{3}+\nabla p_{3}=\vec{f}-\vec{v}_{2 t}, \\
\operatorname{div} \vec{v}_{3}=0, \quad x \in \mathbb{R}_{+}^{n}, \quad t \in(0, T), \\
\left.\vec{v}_{3}\right|_{t=0}=0,\left.\quad \vec{v}_{3}\right|_{x_{n}=0}=0 .
\end{gathered}
$$

Ясно, что функции (2.17), (2.18) действительно являются решением задачи (2.16). Переходим к их оценке.

ПРЕДЛОЖЕНИЕ 2.2. Функция (2.19) удовлетворяет неравенству

$$
\left\|\vec{v}_{1}\right\|_{\dot{W}_{q, r}^{2,1}\left(\Pi_{+}^{T}\right)} \leqslant c\left\|\vec{v}_{0}^{(-)}\right\|_{\dot{B}_{q, r}^{2-2 / r}\left(\mathbb{R}^{n}\right)},
$$

$2 \partial e$

$$
\|f\|_{\dot{B}_{q, r}^{2-2 / r}\left(\mathbb{R}^{n}\right)}=\left(\int_{\mathbb{R}^{n}} \frac{d z}{|z|^{n+2 r-2}}\left(\int_{\mathbb{R}^{n}}|f(x+z)-2 f(x)+f(x-z)|^{q} d x\right)^{r / q}\right)^{1 / r}
$$
- главная часть нормы $\|f\|_{\dot{B}_{q, r}^{2-2 / r}\left(\mathbb{R}^{n}\right)}$, а для решения задачи (2.20) справедлива
оченка

$$
\left\|\vec{v}_{2}\right\|_{\dot{W}_{q, r}^{2,1}\left(\Pi_{+}^{T}\right)} \leqslant c\left(\|\nabla g\|_{L_{q, r}\left(\Pi_{T}^{+}\right)}+\left\|g_{t}\right\|_{L_{r}\left(0, T ; \dot{W}_{q}^{-1}\left(\mathbb{R}_{+}^{n}\right)\right)}\right) .
$$

ДокАЗАТЕЛЬСТво. Пусть $\vec{v}_{1}^{\prime}$ - любая из производных $\vec{v}_{1 t}, \frac{\partial^{2} \vec{v}_{1}}{\partial x_{i} \partial x_{j}}$. Так как соответствуюшая производная $\Gamma^{\prime}(z, t)$ ядра $\Gamma$ четна по $z$, то

$$
\vec{v}_{1}^{\prime}(x, t)=\frac{1}{2} \int_{\mathbb{R}^{n}} \Gamma^{\prime}(z, t) \Delta^{2}(z) \vec{v}_{0}^{(-)}(x) d x
$$

где $\Delta^{2}(z) \vec{v}_{0}^{(-)}(x)=\vec{v}_{0}^{(-)}(x-z)-2 \vec{v}_{0}^{(-)}(x)+\vec{v}_{0}^{(-)}(x+z)$. Отсюда следует

$$
\begin{aligned}
\left\|\vec{v}_{1}^{\prime}(\cdot t)\right\|_{L_{q}\left(\mathbb{R}_{+}^{n}\right)} & \leqslant \frac{1}{2} \int_{\mathbb{R}^{n}}\left|\Gamma^{\prime}(z, t)\right|\left\|\Delta^{2}(z) \vec{v}_{0}^{(-)}(\cdot)\right\|_{L_{q}\left(\mathbb{R}^{n}\right)} d z \\
& \leqslant \frac{c}{t^{1 / r^{\prime}}}\left(\int_{\mathbb{R}^{n}}\left|\Gamma^{\prime}(z, t)\right|\left\|\Delta^{2}(z) \vec{v}_{0}^{(-)}(\cdot)\right\|_{L_{q}\left(\mathbb{R}^{n}\right)}^{r} d z\right)^{1 / r}
\end{aligned}
$$




$$
\begin{aligned}
\left\|\vec{v}_{1}^{\prime}(\cdot t)\right\|_{L_{q, r}\left(\Pi_{+}^{T}\right)}^{r} & \leqslant c \int_{\mathbb{R}^{n}}\left\|\Delta^{2}(z) \vec{v}_{0}^{(-)}(\cdot)\right\|_{L_{q}\left(\mathbb{R}^{n}\right)}^{r} d z \int_{0}^{T}\left|\Gamma^{\prime}(z, t)\right| \frac{d t}{t^{r-1}} \\
& \leqslant c \int_{\mathbb{R}^{n}}\left\|\Delta^{2}(z) \vec{v}_{0}^{(-)}(\cdot)\right\|_{L_{q}\left(\mathbb{R}^{n}\right)}^{r} \frac{d z}{|z|^{n+2 r-2}},
\end{aligned}
$$

и неравенство (2.22) доказано.

Переходим к доказательству (2.23). Хорошо известно, что

$$
\left\|\vec{v}_{2}(\cdot t)\right\|_{\dot{W}_{q}^{2}\left(\mathbb{R}_{+}^{n}\right)}+\left\|\nabla p_{2}(\cdot t)\right\|_{L_{q}\left(\mathbb{R}_{+}^{n}\right)} \leqslant c\|\nabla g\|_{L_{q}\left(\mathbb{R}_{+}^{n}\right)} .
$$

Для оценки $\vec{v}_{2 t}$ рассмотрим вспомогательную задачу

$$
-\nu \Delta \vec{\varphi}+\nabla \pi=\vec{\psi}, \quad \operatorname{div} \vec{\varphi}=0, \quad x \in \mathbb{R}_{+}^{n},\left.\quad \vec{\varphi}\right|_{x_{n}=0}=0,
$$

где $\vec{\psi}$ - произвольное гладкое финитное векторное поле в $\mathbb{R}_{+}^{n}$. Интегрируя по частям, получаем

$$
\int_{\mathbb{R}_{+}^{n}} \vec{v}_{2 t} \cdot \vec{\psi} d x=\int_{\mathbb{R}_{+}^{n}} \vec{v}_{2 t} \cdot(-\nu \Delta \vec{\varphi}+\nabla \pi) d x=\int_{\mathbb{R}_{+}^{n}} g_{t} \pi d x
$$

и, следовательно,

$$
\left|\int_{\mathbb{R}_{+}^{n}} \vec{v}_{2 t} \cdot \vec{\psi} d x\right| \leqslant\left\|g_{t}(\cdot t)\right\|_{\dot{W}_{q}^{-1}\left(\mathbb{R}_{+}^{n}\right)}\|\nabla \pi\|_{L_{q^{\prime}}\left(\mathbb{R}_{+}^{n}\right)} \leqslant c\left\|g_{t}(\cdot t)\right\|_{\dot{W}_{q}^{-1}\left(\mathbb{R}_{+}^{n}\right)}\|\vec{\psi}\|_{L_{q^{\prime}}\left(\mathbb{R}_{+}^{n}\right)} \cdot
$$

В силу произвольности $\vec{\psi}$ это неравенство влечет за собой

$$
\left\|\vec{v}_{2 t}\right\|_{L_{q, r}\left(\Pi_{+}^{T}\right)} \leqslant c\left\|g_{t}\right\|_{L_{r}\left(0, T ; W_{q}^{-1}\left(\mathbb{R}_{+}^{n}\right)\right)} \cdot
$$

Отсюда и из (2.24) вытекает (2.23). Предложение доказано.

Предложения 2.1 и 2.2 приводят к следующему результату.

Tеорема 2.1. Пусть $\vec{f} \in L_{q, r}\left(\Pi_{+}^{T}\right), \nabla g \in L_{q, r}\left(\Pi_{+}^{T}\right)$, существует производная $g_{t} \in L_{r}\left(0, T ; W_{q}^{-1}\left(\mathbb{R}_{+}^{n}\right)\right), g \in L_{q, r}(K \times(0, T))$ для любого компакта $K \subset \overline{\mathbb{R}}_{+}^{n}$, а $\vec{v}_{0}$ обладает тем свойством, что $\vec{v}_{0}^{(-)} \in \dot{B}_{q, r}^{2-2 / r}\left(\mathbb{R}^{n}\right)$. Предположиим, кроме того, что выполнено (в слабом смысле) условие согласования $\operatorname{div} \vec{v}_{0}(x, 0)=g(x, 0)$. Тогда задача (2.16) имеет решение $(\vec{v}, \nabla p)$, удовлетворяющее неравенству

$$
\begin{aligned}
& \|\vec{v}\|_{\dot{W}_{q, r}^{2,1}\left(\Pi_{+}^{T}\right)}+\|\nabla p\|_{L_{q, r}\left(\Pi_{+}^{T}\right)} \\
& \quad \leqslant c\left(\|\vec{f}\|_{L_{q, r}\left(\Pi_{+}^{T}\right)}+\|\nabla g\|_{L_{q, r}\left(\Pi_{T}^{+}\right)}+\left\|g_{t}\right\|_{L_{r}\left(0, T ; W_{q}^{-1}\left(\mathbb{R}_{+}^{n}\right)\right)}+\left\|\vec{v}_{0}^{(-)}\right\|_{\dot{B}_{q, r}^{2-2 / r}\left(\mathbb{R}^{n}\right)}\right)
\end{aligned}
$$


с постоянной, не зависящей от T. Решение единственно в классе функций $\vec{v} \in$ $\dot{W}_{q, r}^{2,1}\left(\Pi_{+}^{T}\right), \nabla p \in L_{q, r}\left(\Pi_{+}^{T}\right)$, maкux, чmo $\vec{v} \in L_{q, r}(K \times(0, T]), \forall K \subset \overline{\mathbb{R}}_{+}^{n}$.

Действительно, сушествование решения и оценка (2.25) следуют из проведенных вьше построений и предложений 2.1, 2.2, а единственность можно установить с помошью соображений дуальности: разность $\vec{w}=\vec{v}^{(1)}-\vec{v}^{(2)}$ двух решений из указанного класса принадлежит $L_{q, r}\left(\Pi_{+}^{T^{\prime}}\right)$ при любом $T^{\prime}<T$ и удовлетворяет соотношению

$$
\int_{0}^{T^{\prime}} \int_{\mathbb{R}^{n}} \vec{w}\left(-\vec{\varphi}_{t}-\nu \Delta \vec{\varphi}+\nabla \pi\right) d x d t=0
$$

при любых финитных $\vec{\varphi}, \pi,\left.\vec{\varphi}\right|_{t=T^{\prime}}=0$. Так как множество $-\vec{\varphi}_{t}-\nu \Delta \vec{\varphi}+\nabla \pi$ плотно в $L_{q^{\prime}, r^{\prime}}\left(\Pi_{+}^{T^{\prime}}\right)$, то $\vec{w}=0$.

Отметим, что условие $\vec{v}_{0}^{(-)} \in \dot{B}_{q, r}^{2-2 / r}\left(\mathbb{R}^{n}\right)$ выделяет из класса $\dot{B}_{q, r}^{2-2 / r}\left(\mathbb{R}^{n}\right)$ элементы, согласованные с нулем на границе $\left\{x_{n}=0\right\}$ (см. по этому поводу [10], где доказана и оценка (2.22)).

Наконец, сформулируем аналогичную теорему для задачи Коши

$$
\begin{gathered}
\vec{v}_{t}-\nu \Delta \vec{v}+\nabla p=\vec{f} \\
\operatorname{div} \vec{v}=g(x, t), \quad x \in \mathbb{R}^{n}, \quad t \in[0, T), \\
\left.\vec{v}\right|_{t=0}=\vec{v}_{0}
\end{gathered}
$$

Teоpema 2.2. Пусть $\vec{f} \in L_{q, r}\left(\Pi^{T}\right), \Pi^{T}=\mathbb{R}^{n} \times(0, T), \nabla g \in L_{q, r}\left(\Pi^{T}\right), g_{t} \in$ $L_{r}\left(0, T ; \dot{W}_{q}^{-1}\left(\mathbb{R}^{n}\right)\right), \vec{v}_{0} \in \dot{B}_{q, r}^{2-2 / r}\left(\mathbb{R}^{n}\right), g \in L_{q, r}(K \times(0, T)), \vec{v}_{0} \in L_{q}(K), \forall K \subset \mathbb{R}^{n}$, $u \operatorname{div} \vec{v}_{0}=g(x, 0)$. Тогда задача (2.26) имеет решение такое, что

$$
\begin{aligned}
& \|\vec{v}\|_{\dot{W}_{q, r}^{2,1}\left(\Pi^{T}\right)}+\|\nabla p\|_{L_{q, r}\left(\Pi^{T}\right)} \\
& \quad \leqslant c\left(\|\vec{f}\|_{L_{q, r}\left(\Pi^{T}\right)}+\|\nabla g\|_{L_{q, r}\left(\Pi^{T}\right)}+\left\|g_{t}\right\|_{L_{r}\left(0, T ; W_{q}^{-1}\left(\mathbb{R}^{n}\right)\right)}+\left\|\vec{v}_{0}\right\|_{\dot{B}_{q, r}^{2-2 / r}\left(\mathbb{R}^{n}\right)}\right) .
\end{aligned}
$$

Решение единственно в классе функций со следующими свойствами:

$$
\vec{v} \in \dot{W}_{q, r}^{2,1}\left(\mathbb{R}^{n}\right), \quad \nabla p \in L_{q, r}\left(\mathbb{R}^{n}\right), \quad \vec{v} \in L_{q, r}(K \times(0, T)), \quad \forall K \subset \mathbb{R}^{n}
$$

Ограничимся указанием на то, что при гладких убьваюших на бесконечности $\vec{f}, \vec{v}_{0}$, $g$ решение имеет вид $(2.17),(2.18)$, где

$$
\vec{v}_{1}(x, t)=\int_{\mathbb{R}^{n}} \Gamma(x-y, t) \vec{v}_{0}(y) d y,
$$

$\left(\vec{v}_{2}, p_{2}\right)$ - решение стационарной системы

$$
-\nu \Delta \vec{v}_{2}+\nabla p_{2}=0, \quad \operatorname{div} \vec{v}_{2}=g-\operatorname{div} \vec{v}_{1}
$$


T.e.

$$
\vec{v}_{2}(x, t)=\int_{\mathbb{R}^{n}} \nabla E(x-y)\left(g(y, t)-\operatorname{div} \vec{v}_{1}(y, t)\right) d y, \quad p_{2}=\nu\left(g-\operatorname{div} \vec{v}_{1}\right),
$$

а $\left(\vec{v}_{3}, p_{3}\right)$ - решение задачи Коши

$$
\begin{gathered}
\vec{v}_{3 t}-\nu \Delta \vec{v}_{3}+\nabla p_{3}=\vec{f}-\vec{v}_{2 t} \equiv \vec{f}_{1}, \quad \operatorname{div} \vec{v}_{3}=0, \quad x \in \mathbb{R}^{n}, \quad t \in(0, T) \\
\left.\vec{v}_{3}\right|_{t=0}=0 .
\end{gathered}
$$

Оно имеет вид

$$
\begin{aligned}
& \vec{v}_{3}(x, t)=\int_{0}^{t} \int_{\mathbb{R}^{n}} \mathbb{T}(x-y, t-\tau) \vec{f}_{1}(y, \tau) d y d \tau \\
& p_{3}(x, t)=\int_{\mathbb{R}^{n}} \nabla E(x-y) \cdot \vec{f}_{1}(y, t) d y
\end{aligned}
$$

где $\mathbb{T}$ - фундаментальная матрица нестационарной системы Стокса с элементами

$$
T_{i j}(x, t)=\delta_{i j} \Gamma(x, t)-\frac{\partial^{2}}{\partial x_{i} \partial x_{j}} \int_{\mathbb{R}^{n}} \Gamma(x-z, t) E(z) d z, \quad i, j=1, \ldots, n
$$

(тензор Озеена). Функции $\left(\vec{v}_{2}, p_{2}\right)$ и $\left(\vec{v}_{3}, p_{3}\right)$ оцениваются так же, как в предложенияx $2.1,2.2$.

2.3. Оценки матрицы Грина. Выведем поточечные оценки матрищы Грина $G_{i j}$ (не используемые в доказательстве оценки (2.25)). Они являются следствием следующего вспомогательного предложения, доказанного в работе [22].

ПРЕДЛОЖЕНИЕ 2.3. Пусть $M(x, t)-$ функиия, заданная $п р и x \in \mathbb{R}_{+}^{n}, t>0, u$ обладающая свойствами

$$
\begin{gathered}
M\left(\lambda x, \lambda^{2} t\right)=\lambda^{m} M(x, t), \quad \forall \lambda>0, \\
\left|D_{x}^{k} D_{t}^{s} M(x, t)\right| \leqslant c t^{\frac{m-|k|-2 s}{s}} \exp \left(-c \frac{|x|^{2}}{t}\right) .
\end{gathered}
$$

Тогда интеграл

$$
J\left(x, y_{n}, t\right)=\int_{\mathbb{R}^{n-1}} E(y) M\left(x^{\prime}-y^{\prime}, x_{n}, t\right) d y^{\prime}
$$

удовлетворяет условиям

$$
\begin{gathered}
J\left(\lambda x, \lambda y_{n}, \lambda^{2} t\right)=\lambda^{m+1} J\left(x, y_{n}, t\right), \\
\left|D_{x}^{k} D_{y_{n}}^{l} D_{t}^{s} J\left(x, y_{n}, t\right)\right| \leqslant c t^{\frac{m+n-1-2 s-k_{n}}{2}}\left[\left|x^{\prime}\right|^{2}+\left(x_{n}+y_{n}\right)^{2}+t\right]^{-\frac{\left|k^{\prime}\right|+l+n-2}{2}} e^{-\frac{c x_{n}^{2}}{t}}
\end{gathered}
$$

əде $x^{\prime}=\left(x_{1}, \ldots, x_{n-1}\right), k^{\prime}=\left(k_{1}, \ldots, k_{n-1}\right)$. 
ДоКАЗАТЕЛЬСТвО. Условие однородности (2.29) легко вытекает из (2.28), а из него следует, что неравенство (2.30) достаточно доказать при $t=1$; кроме того, так как функция $E$ гармоническая, то достаточно ограничиться рассмотрением случаев $l=0, l=1$. Положим $M_{s}(x)=\left.D_{t}^{s} M(x, t)\right|_{t=1}, E_{l}(y)=\left(\frac{\partial}{\partial y_{n}}\right)^{l} E(y), l=0,1$, и оценим производную $D_{x}^{k} J_{l, s}$ интеграла

$$
\int_{\mathbb{R}^{n-1}} E_{l}(y) M_{s}\left(x^{\prime}-y^{\prime}, x_{n}\right) d y^{\prime}=J_{l, s}\left(x, y_{n}\right) .
$$

Имеем

$$
\begin{aligned}
\left|D_{x}^{k} J_{l, s}\left(x, y_{n}\right)\right| & \leqslant c \int_{\mathbb{R}^{n-1}}\left|E_{l}(y)\right| e^{-c\left|x^{\prime}-y^{\prime}\right|^{2}} d y^{\prime} e^{-c x_{n}^{2}} \\
& \leqslant c\left(\int_{\left|y^{\prime}\right| \leqslant 1}\left|E_{l}(y)\right| d y^{\prime}+\int_{\left|y^{\prime}\right| \geqslant 1} e^{-c\left|x^{\prime}-y^{\prime}\right|^{2}} d y^{\prime}\right) e^{-c x_{n}^{2}} \leqslant c e^{-c x_{n}^{2}}
\end{aligned}
$$

Кроме того, полагая $\rho=\left(\left|x^{\prime}\right|^{2}+\left(x_{n}+y_{n}\right)^{2}\right)^{1 / 2}$ и интегрируя по частям, получаем

$$
\begin{aligned}
D_{x}^{k} J_{l, s}\left(x, y_{n}\right)= & \int_{\sigma_{\rho}(x)} D_{y^{\prime}}^{k^{\prime}} E_{l}(y) D_{x_{n}}^{k_{n}} M_{s}\left(x^{\prime}-y^{\prime}, x_{n}\right) d y^{\prime} \\
& +\int_{\mathbb{R}^{n-1} \backslash \sigma_{\rho}(x)} E_{l}(y) D_{x}^{k} M_{s}\left(x^{\prime}-y^{\prime}, x_{n}\right) d y^{\prime} \\
& +\sum_{j^{\prime}} \int_{\partial \sigma_{\rho}(x)} D_{y^{\prime}}^{j^{\prime}} E_{l}(y) D_{x^{\prime}}^{m^{\prime}} D_{x_{n}}^{k_{n}} M_{s}\left(x^{\prime}-y^{\prime}, x_{n}\right) n_{i}\left(y^{\prime}\right) d S_{y} \\
= & I_{1}+I_{2}+I_{3},
\end{aligned}
$$

где $\sigma_{\rho}(x)=\left\{y^{\prime} \in \mathbb{R}^{n-1}:\left|x^{\prime}-y^{\prime}\right|^{2}+x_{n}^{2} \leqslant \rho^{2} / 4\right\}$ (это множество пусто, если $x_{n} \geqslant \rho / 2$ ). Так как

$$
|y|=\sqrt{\left|y^{\prime}\right|^{2}+y_{n}^{2}} \geqslant \sqrt{\left|x^{\prime}\right|^{2}+\left(x_{n}+y_{n}\right)^{2}}-\sqrt{\left|x^{\prime}-y^{\prime}\right|^{2}+x_{n}^{2}} \geqslant \frac{\rho}{2}
$$

для $y^{\prime} \in \sigma_{\rho}(x)$, то

$$
\left|I_{1}\right| \leqslant c \rho^{-\left(n-2+l+\left|k^{\prime}\right|\right)} \int_{\sigma_{\rho}(x)} e^{-c\left(\left|x^{\prime}-y^{\prime}\right|^{2}+x_{n}^{2}\right)} d y^{\prime} \leqslant \frac{c e^{-c x_{n}^{2}}}{\rho^{n-2+l+\left|k^{\prime}\right|}} .
$$

Далее,

$$
\left|I_{2}\right| \leqslant c e^{-c \rho^{2}} \int_{\mathbb{R}^{n-1}}\left|E_{l}(y)\right| e^{-c\left|x^{\prime}-y^{\prime}\right|^{2}} d y^{\prime} \leqslant c e^{-c \rho^{2}} .
$$

В третьем члене $I_{3}$ мультииндексы $j^{\prime}$ и $m^{\prime}$ таковы, что $j^{\prime}+m^{\prime}+i^{\prime}=k^{\prime}$, где

$$
i^{\prime}=(\underbrace{0, \ldots, 0,1}_{i}, 0, \ldots, 0)
$$


а $\vec{n}\left(y^{\prime}\right)$ - нормаль к $\sigma_{\rho}(x)$. Поэтому

$$
\left|I_{3}\right| \leqslant c \sum_{j^{\prime}} \rho^{n-2} \rho^{-\left(n-2+l+j^{\prime}\right)} e^{-c \rho^{2}} \leqslant c e^{-c \rho^{2}}\left(1+\frac{1}{\rho^{l+\left|k^{\prime}\right|}}\right) .
$$

Объединяя полученные оценки с (2.31), приходим к неравенству

$$
\left|D_{x}^{k} J_{l, s}(x)\right| \leqslant c e^{-c x_{n}^{2}}\left(\rho^{2}+1\right)^{-\frac{\left|k^{\prime}\right|+l+n-2}{2}},
$$

откуда следует (2.30). Предложение доказано.

Переходим к оценкам функций $G_{i j}$ и $P_{j}$. Следуюший результат вытекает из определения $P_{j}$ и из предложения 2.3 .

ПРЕДЛОЖЕНИЕ 2.4. Справедливы неравенства

$$
\left|\left(\frac{\partial}{\partial t}\right)^{s} D_{x}^{k} D_{z}^{m} P_{j}(x, z, t)\right| \leqslant c t^{-1-s-\frac{m_{n}}{2}}\left(\left|x-z^{*}\right|^{2}+t\right)^{-\frac{n-1+|k|+\left|m^{\prime}\right|}{2}} e^{-\frac{c z_{n}^{2}}{t}} .
$$

Оценка $G_{i j}$ сводится к оценке функций

$$
\begin{aligned}
G_{i j}^{\prime}(x, z, t) & =4\left(1-\delta_{j n}\right) \frac{\partial}{\partial x_{j}} \int_{0}^{x_{n}} \int_{\mathbb{R}^{n-1}} \frac{\partial E(x-y)}{\partial x_{i}} \Gamma\left(y-z^{*}, t\right) d y \\
& =4\left(1-\delta_{j n}\right) \frac{\partial}{\partial x_{j}} \int_{0}^{x_{n}} \int_{\mathbb{R}^{n-1}} E_{i}(y) \Gamma\left(x-z^{*}-y, t\right) d y .
\end{aligned}
$$

ПРЕДЛОЖЕНИЕ 2.5. Функиии $G_{i j}^{\prime}$ удовлетворяют неравенствам

$$
\left|\left(\frac{\partial}{\partial t}\right)^{s} D_{x}^{k} D_{z}^{m} G_{i j}^{\prime}(x, z, t)\right| \leqslant c t^{-s-\frac{m_{n}}{2}}\left(t+x_{n}^{2}\right)^{-k_{n} / 2}\left(\left|x-z^{*}\right|^{2}+t\right)^{-\frac{n+\left|k^{\prime}\right|+\left|m^{\prime}\right|}{2}} e^{-\frac{c z_{n}^{2}}{t}} .
$$

ДокАЗАТЕльСТво. Вследствие предложения 2.3 функция

$$
J_{i}\left(x, z, y_{n}, t\right)=\int_{\mathbb{R}^{n-1}} E_{i}(y) \Gamma\left(x-z^{*}-y, t\right) d y^{\prime}
$$

подчиняется неравенствам

$$
\left|\left(\frac{\partial}{\partial t}\right)^{s} D_{x}^{k} D_{z}^{m} J_{i}\right| \leqslant c t^{-\frac{1}{2}-s-\frac{m_{n}+k_{n}}{2}}\left[\left|x-z^{*}\right|^{2}+t\right]^{-\frac{\left|k^{\prime}\right|+\left|m^{\prime}\right|+n-1}{2}} e^{-\frac{c\left(x_{n}+z_{n}-y_{n}\right)^{2}}{t}} .
$$

Отсюда следует, что

$$
\left|G_{i j}^{\prime}(x, z, t)\right| \leqslant c\left(\left|x-z^{*}\right|^{2}+t\right)^{-n / 2} e^{-\frac{c z_{n}^{2}}{t}}
$$

и, более того,

$$
\left|D_{x^{\prime}}^{k^{\prime}} D_{z}^{m} G_{i j}^{\prime}(x, z, t)\right| \leqslant c t^{-m_{n} / 2}\left(\left|x-z^{*}\right|^{2}+t\right)^{-\frac{n+\left|k^{\prime}\right|+\left|m^{\prime}\right|}{2}} e^{-\frac{c z_{n}^{2}}{t}} .
$$


Подсчитаем производную $\frac{\partial G_{i j}^{\prime}}{\partial x_{n}}$. Если $i \neq n$, то

$$
\begin{aligned}
\frac{\partial}{\partial x_{n}} G_{i j}^{\prime}= & \frac{\partial^{2}}{\partial x_{i} \partial x_{j}} \int_{0}^{x_{n}} \int_{\mathbb{R}^{n-1}} E_{n}(x-y) \Gamma\left(y-z^{*}, t\right) d y \\
& +\frac{\partial^{2}}{\partial x_{i} \partial x_{j}} \int_{\mathbb{R}^{n-1}} E\left(x^{\prime}-y^{\prime}, 0\right) \Gamma\left(y^{\prime}-z^{\prime}, x_{n}+z_{n}, t\right) d y^{\prime}
\end{aligned}
$$

а если $i=n$, то

$$
\frac{\partial G_{n j}^{\prime}}{\partial x_{n}}=-\sum_{\mu=1}^{n-1} \frac{\partial G_{\mu j}^{\prime}}{\partial x_{\mu}}+2 \frac{\Gamma\left(x-z^{*}, t\right)}{\partial x_{j}}
$$

В обоих случаях имеем

$$
\begin{aligned}
\left|\frac{\partial}{\partial x_{n}} D_{x}^{k^{\prime}} D_{z}^{m} G_{i j}^{\prime}\right| \leqslant & c\left(\left|x-z^{*}\right|^{2}+t\right)^{-\frac{n+\left|k^{\prime}\right|+\left|m^{\prime}\right|}{2}} t^{-m_{n} / 2} e^{-\frac{c z_{n}^{2}}{t}} \\
& +c t^{-\frac{1+m_{n}}{2}}\left(\left|x-z^{*}\right|^{2}+t\right)^{-\frac{n+\left|k^{\prime}\right|+\left|m^{\prime}\right|+1}{2}} e^{-\frac{c\left(x_{n}+z_{n}\right)^{2}}{t}} \\
\leqslant & c\left(t+x_{n}^{2}\right)^{-1 / 2} t^{-m_{n} / 2}\left(\left|x-z^{*}\right|^{2}+t\right)^{-n / 2} e^{-\frac{c z_{n}^{2}}{t}} .
\end{aligned}
$$

Дифференцируя формулы (2.35) и (2.36) по $x_{n}$ и применяя несколько раз предложение 2.3 , нетрудно получить оценку $(2.34)$ в случае $s=0$. Для оценки производной $\frac{\partial G_{i j}^{\prime}}{\partial t}$ воспользуемся соотношением

$$
\frac{\partial G_{i j}^{\prime}}{\partial t}=\nu \Delta G_{i j}^{\prime}-\frac{\partial P_{j}}{\partial x_{i}}
$$

Оно позволяет вьвести из (2.32) и из уже доказанного неравенства (2.34) с $s=0$ то же неравенство с $s=1$. Дифференцируя (2.37) по $t$, получаем (2.34) в общем случае. Предложение доказано.

Так как функция $\Gamma\left(x-z^{*}, t\right)$ также удовлетворяет неравенству (2.34), мы можем записать $G_{i j}(x, z, t)$ в виде

$$
G_{i j}(x, z, t)=-\delta_{i j} \Gamma(x-z, t)+G_{i j}^{*}(x, z, t), \quad G_{i j}^{*}=\delta_{i j} \Gamma\left(x-z^{*}, t\right)+G_{i j}^{\prime} ;
$$

в силу (2.34)

$$
\left|\left(\frac{\partial}{\partial t}\right)^{s} D_{x}^{k} D_{z}^{m} G_{i j}^{*}(x, z, t)\right| \leqslant c t^{-s-m_{n} / 2}\left(t+x_{n}^{2}\right)^{-k_{n} / 2}\left(\left|x-z^{*}\right|^{2}+t\right)^{-\frac{n+\left|k^{\prime}\right|+\left|m^{\prime}\right|}{2}} e^{-\frac{c z_{n}^{2}}{t}} .
$$

Следствием (2.38) является оценка

$$
\begin{aligned}
\int_{\mathbb{R}_{+}^{n}}\left|G_{i j}(x, z, t)\right| d z & \leqslant \delta_{i j}+c \int_{\mathbb{R}_{+}^{n}}\left(\left|x-z^{*}\right|^{2}+t\right)^{-n / 2} e^{-\frac{c z_{n}^{2}}{t}} d t \\
& \leqslant \delta_{i j}+c \int_{0}^{\infty}\left(z_{n}^{2}+t\right)^{-1 / 2} e^{-\frac{c z_{n}^{2}}{t}} d z_{n} \leqslant c,
\end{aligned}
$$

справедливая при любом $t>0$. 
Полученные оценки элементов матрицы $Г$ рина $G_{i j}$ позволяют построить решение задачи (2.10) при любом ограниченном непрерьвном $\vec{v}_{0}(x)$. Векторное поле скоростей определяется формулой $\left(2.11_{1}\right)$; оно непрерьвно, и вследствие $(2.39)$

$$
|\vec{v}(x, t)| \leqslant c \sup _{\mathbb{R}_{+}^{n}}\left|\vec{v}_{0}(y)\right|
$$

а для производных $\vec{v}$ имеем оценки

$$
\left|\left(\frac{\partial}{\partial t}\right)^{s} D_{x}^{k} \vec{v}(x, t)\right| \leqslant c \sup _{\mathbb{R}_{+}^{n}}\left|\vec{v}_{0}(y)\right| t^{-s-|k| / 2} .
$$

Формула для давления $\left(2.11_{2}\right)$ теряет смысл, так как оценка $(2.32)$ с $s=0, k=0$, $m=0$ не гарантирует сходимости интеграла в $\left(2.11_{2}\right)$ при любом ограниченном $\vec{v}_{0}(y)$. Но для градиента давления имеем обычное представление

$$
\nabla p(x, t)=\sum_{j=1}^{n-1} \int_{\mathbb{R}_{+}^{n}} \nabla P_{j}(x, y, t) v_{0 j}(y) d y
$$

причем вследствие (2.32)

$$
\begin{aligned}
|\nabla p(x, t)| & \leqslant c \sup \left|\vec{v}_{0}(y)\right| \int_{\mathbb{R}_{+}^{n}} t^{-1}\left(\left|x-z^{*}\right|^{2}+t\right)^{-n / 2} e^{-\frac{c z_{n}^{2}}{t}} d z \\
& \leqslant c \sup \left|\vec{v}_{0}(y)\right| t^{-1 / 2}\left(x_{n}^{2}+t\right)^{-1 / 2} \underset{x_{n} \rightarrow \infty}{\longrightarrow} 0 .
\end{aligned}
$$

Можно показать, что построенное решение задачи (2.10) с ограниченным непрерьвным $\vec{v}_{0}$ единственно в классе функщий, удовлетворяюших условиям (2.40)-(2.42).

\section{§3. Доказательство теорем 1.1, 1.2}

3.1. Случай $\vec{v}_{0}=0$. Сначала положим $\vec{v}_{0}=0$ и рассмотрим задачу

$$
\begin{gathered}
\vec{v}_{t}-\nu \Delta \vec{v}+\nabla p=\vec{f}, \quad \operatorname{div} \vec{v}=0, \quad x \in \Omega, \quad t \in(0, T) \\
\left.\vec{v}\right|_{t=0}=0,\left.\quad \vec{v}\right|_{x \in S}=0
\end{gathered}
$$

в ограниченной или внешней области $\Omega \subset \mathbb{R}^{n}$. Ради некоторых технических упрощений будем считать, что $\vec{f}$ удовлетворяет условиям

$$
\operatorname{div} \vec{f}=0,\left.\quad \vec{f} \cdot \vec{n}\right|_{x \in S}=0
$$

(т.е. $\int_{\Omega} \vec{f} \cdot \nabla \varphi d x=0$ для любой гладкой финитной $\varphi$ ). Как и в случае полупространства, это предположение не является ограничением обшности. Получим сначала априорную оценку любого решения $\vec{v} \in W_{q, r}^{2,1}\left(Q^{T}\right), \nabla p \in L_{q, r}\left(Q^{T}\right)$ задачи. Оценим 
решение в окрестности любой точки $x_{0} \in S$. Поместим начало координат в $x_{0}$, повернем оси так, чтобы ось $x_{n}$ была направлена вдоль внутренней нормали к $S$ в точке $x_{0}$, и введем функции

$$
\vec{v}^{\prime}(x, t)=\vec{v}(x, t) \zeta_{\lambda}(x), \quad p^{\prime}(x, t)=p(x, t) \zeta_{\lambda}(x)
$$

где $\zeta_{\lambda}(x)=\zeta\left(\left(x-x_{0}\right) / \lambda\right), \zeta(z)-$ стандартная срезающая функция из $C_{0}^{\infty}\left(\mathbb{R}^{n}\right)$, равная единице при $|z|<1 / 2$ и нулю при $|z|>1$ и являюшаяся монотонной функцией $|z|$. Введем новые координаты $y=U^{-1} x$ :

$$
y^{\prime}=x^{\prime}, \quad y_{n}=x_{n}-F\left(x^{\prime}\right)
$$

где $x^{\prime}=\left(x_{1}, \ldots, x_{n-1}\right)$ и $F-$ функция, задающая поверхность $S$ вблизи $x_{0}=0$ посредством уравнения $y_{n}=F\left(y^{\prime}\right),\left|y^{\prime}\right| \leqslant d$. Положим $\vec{v}^{\prime}(U z, t)=\vec{u}(z, t), p^{\prime}(U z, t)=s(z, t)$, $\widetilde{\zeta}_{\lambda}(U z)=\zeta_{\lambda}(z)$ и будем считать эти функции заданньми во всем полупространстве $\mathbb{R}_{+}^{n}$ и равными нулю вне носителя $\widetilde{\zeta}_{\lambda}$. Ясно, что $\vec{u}, s$ удовлетворяют соотношениям

$$
\begin{gathered}
\vec{v}_{t}-\nu \nabla^{\prime 2} \vec{u}+\nabla^{\prime} s=\vec{f}^{\prime} \widetilde{\zeta}_{\lambda}+\vec{f}_{1}(z, t), \\
\nabla^{\prime} \cdot \vec{u}=\vec{v}^{\prime} \cdot \nabla^{\prime} \widetilde{\zeta}_{\lambda}, \quad z \in \mathbb{R}_{+}^{n}, \quad t \in[0, T], \\
\left.\vec{u}\right|_{t=0}=0,\left.\quad \vec{u}\right|_{z_{n}=0}=0,
\end{gathered}
$$

где

$$
\nabla^{\prime}=\left(\frac{\partial}{\partial z_{1}}-F_{1}^{\prime} \frac{\partial}{\partial z_{n}}, \ldots, \frac{\partial}{\partial z_{n-1}}-F_{n-1}^{\prime} \frac{\partial}{\partial z_{n}}, \frac{\partial}{\partial z_{n}}\right), \quad F_{j}^{\prime}=\frac{\partial F}{\partial z_{j}}
$$

и

$$
\vec{f}_{1}=s \nabla^{\prime} \widetilde{\zeta}_{\lambda}-\nu \nabla^{\prime} \vec{u}\left(\nabla^{\prime} \widetilde{\zeta}_{\lambda}\right)-\nu \vec{u} \nabla^{\prime 2} \widetilde{\zeta}_{\lambda}
$$

Запишем (3.4) в виде

$$
\begin{gathered}
\vec{u}_{t}-\nu \Delta \vec{u}+\nabla s=\vec{f} \zeta_{\lambda}+\vec{f}_{1}+\vec{f}_{2} \\
\operatorname{div} \vec{u}=\vec{v}^{\prime} \cdot \nabla^{\prime} \widetilde{\zeta}_{\lambda}+\sum_{j=1}^{n-1} F_{j} \frac{\partial u_{j}}{\partial z_{n}} \equiv g \\
\left.\vec{u}\right|_{t=0}=0,\left.\quad \vec{u}\right|_{z_{n}=0}=0
\end{gathered}
$$

c $\overrightarrow{f_{2}}=\nu\left(\nabla^{\prime 2}-\nabla^{2}\right) \vec{u}+\left(\nabla-\nabla^{\prime}\right) s$.

Воспользуемся оценкой (2.12) для задачи (2.4), убедившись предварительно, что $g_{t} \in L_{r}\left(0, T ; \dot{W}_{q}^{-1}\left(\mathbb{R}_{+}^{n}\right)\right)$. Для любой $\varphi \in \dot{W}_{q^{\prime}}^{1}\left(\mathbb{R}_{+}^{n}\right)$ имеем

$$
\int_{\mathbb{R}_{+}^{n}} g \varphi d z=\int_{\mathbb{R}_{+}^{n}}\left(\vec{v}^{\prime} \cdot \nabla^{\prime} \widetilde{\zeta}_{\lambda}(\varphi-\bar{\varphi})-\sum_{j=1}^{n-1} F_{j} u_{j} \frac{\partial \varphi}{\partial z_{n}}\right) d z
$$


где $\bar{\varphi}=\frac{1}{|B(\lambda)|} \int_{B(\lambda)} \varphi d z$ и $B(\lambda)-$ полушар $\left\{|z| \leqslant c \lambda, z_{n}>0\right\}$, содержаший $\operatorname{supp} \widetilde{\zeta}_{\lambda}$. Отсюда следует

$$
\begin{aligned}
\int_{\mathbb{R}_{+}^{n}} g_{t} \varphi d z= & \int_{\mathbb{R}_{+}^{n}}\left(\left(\nu \nabla^{\prime 2} \vec{v}^{\prime}-\nabla^{\prime} p^{\prime}+\vec{f}^{\prime}\right) \cdot \nabla^{\prime} \widetilde{\zeta}_{\lambda}(\varphi-\bar{\varphi})-\sum_{j=1}^{n-1} F_{j} u_{j t} \frac{\partial \varphi}{\partial z_{n}}\right) d z \\
= & \int_{\mathbb{R}_{+}^{n}}\left\{-\nu \sum_{k=1}^{n} \nabla_{k}^{\prime} \vec{v}^{\prime} \cdot \nabla_{k}^{\prime}\left(\nabla^{\prime} \widetilde{\zeta}_{\lambda}(\varphi-\bar{\varphi})\right)+p^{\prime} \nabla^{\prime} \cdot\left(\nabla \widetilde{\zeta}_{\lambda}(\varphi-\bar{\varphi})\right)\right. \\
& \left.+\vec{f} \cdot \nabla^{\prime} \widetilde{\zeta}_{\lambda}(\varphi-\bar{\varphi})-\sum_{j=1}^{n-1} F_{j} u_{j t} \frac{\partial \varphi}{\partial z_{n}}\right\} d z \\
& -\left.\int_{\mathbb{R}^{n-1}} p^{\prime}\left(\sum_{j=1}^{n-1} F_{j} \nabla_{j}^{\prime}-\frac{\partial}{\partial z_{n}}\right) \widetilde{\zeta}_{\lambda}^{\prime}(\varphi-\bar{\varphi}) d z^{\prime}\right|_{z_{n}=0} \\
& +\left.\int_{\mathbb{R}^{n-1}}\left(\sum_{j=1}^{n-1} F_{j} \nabla_{j}^{\prime}-\frac{\partial}{\partial z_{n}}\right) \vec{v} \cdot \nabla^{\prime} \widetilde{\zeta}_{\lambda}^{\prime}(\varphi-\bar{\varphi}) d z^{\prime}\right|_{z_{n}=0} .
\end{aligned}
$$

Оценивая $\varphi-\bar{\varphi}$ с помощью неравенства Пуанкаре, получаем

$$
\begin{aligned}
\left|\int_{\mathbb{R}_{+}^{n}} g_{t} \varphi d x\right| \leqslant & c\|\nabla \varphi\|_{L_{q^{\prime}}\left(\mathbb{R}_{+}^{n}\right)}\left(\lambda^{-1}\left\|\nabla \vec{v}^{\prime}\right\|_{L_{q}(B(\lambda))}+\lambda^{-1}\left\|p^{\prime}\right\|_{L_{q}(B(\lambda))}\right. \\
& +\lambda^{-1+1 / q}\left\|\nabla \vec{v}^{\prime}\right\|_{L_{q^{\prime}}(b(\lambda))}+\lambda^{-1+1 / q}\left\|p^{\prime}\right\|_{L_{q}(b(\lambda))} \\
& \left.+\|\vec{f}\|_{L_{q^{\prime}}(B(\lambda))}+\max _{B(\lambda)}\left|\nabla^{\prime} F\right|\left\|\vec{u}_{t}\right\|_{L_{q}(B(\lambda))}\right)
\end{aligned}
$$

где $b(\lambda)$ - плоская часть границы $B(\lambda)$. Так как $S \in C^{2}$, то $\max _{B(\lambda)}\left|\nabla^{\prime} F\right| \leqslant c \lambda$, так что

$$
\begin{aligned}
\left\|g_{t}(\cdot, t)\right\|_{\dot{W}_{q}^{1}\left(\mathbb{R}_{+}^{n}\right)} \leqslant & c\left(\lambda^{-1}\left\|\nabla \vec{v}^{\prime}\right\|_{L_{q}(B(\lambda))}+\lambda^{-1+1 / q}\left\|\nabla \vec{v}^{\prime}\right\|_{L_{q}(b(\lambda))}\right. \\
& +\lambda^{-1}\left\|p^{\prime}\right\|_{L_{q}(B(\lambda))}+\lambda^{-1+1 / q}\left\|p^{\prime}\right\|_{L_{q}(b(\lambda))} \\
& \left.+\|\vec{f}\|_{L_{q, r}(B(\lambda))}+\lambda\left\|\vec{u}_{t}\right\|_{L_{q}(B(\lambda))}\right) .
\end{aligned}
$$

Теперь, пользуясь оценкой (2.25), получаем при достаточно малом $\lambda$

$$
\begin{aligned}
& \|\vec{u}\|_{\dot{W}_{q, r}^{2,1}\left(\Pi_{+}^{T}\right)}+\|\nabla q\|_{L_{q, r}\left(\Pi_{+}^{T}\right)} \\
& \leqslant c\left(\|\vec{f}\|_{L_{q, r}(B(\lambda) \times(0, T))}+\lambda^{-1}\left\|\nabla \vec{v}^{\prime}\right\|_{L_{q, r}(B(\lambda) \times(0, T))}+\lambda^{-1}\left\|p^{\prime}\right\|_{L_{q, r}(B(\lambda) \times(0, T))}\right. \\
& \quad+\lambda^{-1+1 / q}\left(\left\|p^{\prime}\right\|_{L_{q, r}(b(\lambda) \times(0, T))}+\left\|\nabla \vec{v}^{\prime}\right\|_{L_{q, r}(b(\lambda) \times(0, T))}\right) \\
& \left.\quad+\lambda^{-2}\left\|\vec{v}^{\prime}\right\|_{L_{q, r}(B(\lambda) \times(0, T))}\right) .
\end{aligned}
$$

Это неравенство справедливо в $\lambda$-окрестности любой точки $S$. Выбрав конечную достаточно густую сеть таких точек $x^{(k)}$, мы покроем их окрестностями пограничную 
полоску $S$ ширины $\lambda / 4$. Остается оценить решения во внутренней подобласти $\Omega_{\lambda} \subset \Omega$, точки которой отстоят от $S$ не менее чем на $\lambda / 4$. Введем снова функции $(3.3)$, понимая теперь под $\zeta_{\lambda}$ функцию, равную единице в $\Omega_{\lambda}$ и нулю в $\Omega \backslash \Omega_{\lambda / 2}$. Будем считать $\vec{v}^{\prime}$ и $p^{\prime}$ равными нулю в $\mathbb{R}^{n} \backslash \Omega$ и рассмотрим их как решение задачи Коши

$$
\begin{gathered}
\vec{v}_{t}^{\prime}-\nu \Delta \vec{v}^{\prime}+\nabla p^{\prime}=\vec{f} \zeta_{\lambda}+\vec{f}_{1}, \quad \operatorname{div} \vec{v}^{\prime}=\nabla \zeta_{\lambda} \cdot \vec{v}, \quad x \in \mathbb{R}^{n}, \\
\left.\vec{v}^{\prime}\right|_{t=0}=0
\end{gathered}
$$

c $\vec{f}_{1}=p \nabla \zeta_{\lambda}-\nu \nabla \vec{v}\left(\nabla \zeta_{\lambda}\right)-\nu \vec{v} \nabla^{2} \zeta_{\lambda}$.

Повторив проведенное вьше рассуждение (но использовав (2.27) вместо (2.25)), придем к неравенству

$$
\begin{aligned}
& \left\|\vec{v}^{\prime}\right\|_{\dot{W}_{q, r}^{2,1}\left(\Pi^{T}\right)}+\left\|\nabla p^{\prime}\right\|_{L_{q, r}\left(\Pi^{T}\right)} \\
& \leqslant c\left(\|\vec{f}\|_{L_{q, r}\left(\Omega_{\lambda / 2} \times(0, T)\right)}+\lambda^{-1}\|\nabla \vec{v}\|_{L_{q, r}(C(\lambda) \times(0, T))}\right. \\
& \quad+\lambda^{-2}\|\vec{v}\|_{L_{q, r}(C(\lambda) \times(0, T))}+\lambda^{-1}\|p\|_{\left.L_{q, r}(C(\lambda) \times(0, T))\right)},
\end{aligned}
$$

где $C(\lambda)=\Omega_{\lambda / 2} \backslash \Omega_{\lambda}$. Сложим эту оценку и неравенство (3.7) при всех $x^{(k)}$ (число этих точек конечно). Это даст

$$
\begin{aligned}
& \|\vec{v}\|_{\dot{W}_{q, r}^{2,1}\left(Q^{T}\right)}+\|\nabla p\|_{L_{q, r}\left(Q^{T}\right)} \\
& \quad \leqslant \quad\left(\|\vec{f}\|_{L_{q, r}\left(Q^{T}\right)}+\|\nabla \vec{v}\|_{L_{q, r}\left(\Omega_{1} \times(0, T)\right)}+\|p\|_{L_{q, r}\left(\Omega_{1} \times(0, T)\right)}\right. \\
& \left.\quad+\|\nabla \vec{v}\|_{L_{q, r}(S \times(0, T))}+\|p\|_{L_{q, r}(S \times(0, T))}+\|\vec{v}\|_{L_{q, r}\left(\Omega_{1} \times(0, T)\right)}\right),
\end{aligned}
$$

где $\Omega_{1}$ - некоторая ограниченная область, прилегающая к границе (так что $\left(\Omega \backslash \Omega_{1}\right) \cap$ $S=\varnothing)$.

Для исключения некоторых норм $p$ и $\nabla \vec{v}$ из правой части (3.8) воспользуемся интерполящионными неравенствами

$$
\begin{aligned}
\|\nabla \vec{v}\|_{L_{q, r}\left(\Omega_{1} \times(0, T)\right)} & \leqslant \theta_{1} \sum_{|j|=2}\left\|D^{j} \vec{v}\right\|_{L_{q, r}\left(\Omega_{1} \times(0, T)\right)}+c \theta_{1}^{-1}\|\vec{v}\|_{L_{q, r}\left(\Omega_{1} \times(0, T)\right)}, \\
\|\nabla \vec{v}\|_{L_{q, r}(S \times(0, T))} & \leqslant c \theta_{2}^{1-1 / q} \sum_{|j|=2}\left\|D^{j} \vec{v}\right\|_{L_{q, r}\left(\Omega_{1} \times(0, T)\right)}+c \theta_{2}^{-1-1 / q}\|\vec{v}\|_{L_{q, r}\left(\Omega_{1} \times(0, T)\right)}, \\
\|p\|_{L_{q, r}(S \times(0, T))} & \leqslant c \theta_{3}^{1-1 / q}\|\nabla p\|_{L_{q, r}\left(\Omega_{1} \times(0, T)\right)}+c \theta_{3}^{-1 / q}\|p\|_{L_{q, r}\left(\Omega_{1} \times(0, T)\right)},
\end{aligned}
$$

справедливьми при сколь угодно малых положительных $\theta_{i}$. Кроме того, примем во внимание, что $p(x, t)$ является решением задачи

$$
\begin{gathered}
\Delta p=0, \quad x \in \Omega, \\
\left.\frac{\partial p}{\partial n}\right|_{x \in S}=\nu \vec{n} \cdot \Delta \vec{v}=\left.\nu \sum_{i, k=1}^{n}\left(n_{k} \frac{\partial}{\partial x_{i}}-n_{i} \frac{\partial}{\partial x_{k}}\right) \frac{\partial v_{k}}{\partial x_{i}}\right|_{x \in S} .
\end{gathered}
$$


Из представления решения этой задачи в виде потенциала простого слоя следует (см. [4]), что

$$
\|p\|_{L_{q, r}(S \times(0, T))} \leqslant c\|\nabla \vec{v}\|_{L_{q, r}(S \times(0, T))} .
$$

Отсюда и из оценок (3.8) и (3.9)-(3.11) с достаточно малыми $\theta_{i}$ вытекает неравенство

$$
\|\vec{v}\|_{\dot{W}_{q, r}^{2,1}\left(Q^{T}\right)}+\|\nabla p\|_{L_{q, r}\left(Q^{T}\right)} \leqslant c_{1}\|\vec{f}\|_{L_{q, r}\left(Q^{T}\right)}+c_{2}\|\vec{v}\|_{L_{q, r}\left(\Omega_{1} \times(0, T)\right)},
$$

в котором постоянные $c_{1}$ и $c_{2}$ не зависят от $T$.

Ясно, что (3.12) справедливо и для любого $t<T$ с теми же самьми постоянными. Отсюда с помошью леммы Гронуолла легко вывести оценку (1.5) или (1.10) (при $\left.\vec{v}_{0}=0, g=0\right)$ с постоянной $c=c(T)$.

Более точный результат может быть получен, если воспользоваться для оценки последнего члена в (3.12) представлением решения задачи (1.1) с $\vec{v}_{0}=0$ через резольвенту оператора Стокса, оценки которой будут приведены ниже в $\S 4$. В случае ограниченной области это приводит к неравенству (1.5) с постоянной, не зависящей от $T$. В случае внешней области оценок резольвенты для доказательства (1.10) не достаточно; это неравенство было доказано различными способами в [7], [10]. Мы не можем привести здесь все детали доказательства.

Приведем доказательство разрешимости задачи (1.1) из работы [11] (единственность решения из рассматриваемого класса является следствием из доказанной вьше априорной оценки). Будем считать, что $\vec{f}(x, t)$ принадлежит плотному в $L_{q, r}\left(Q^{T}\right)$ множеству гладких финитных векторных полей. Хорошо известно [23], что задача (1.1) с $\vec{v}_{0}=0$ имеет единственное обобщенное решение, которое на самом деле принадлежит классу $W_{2}^{2,1}\left(Q^{T}\right)$, а $\nabla p \in L_{2}\left(Q^{T}\right)$. Следуя работам [24], [11], введем усреднение по $t$

$$
\vec{f}_{\rho}(x, t)=\int_{|s|<\rho} \omega_{\rho}(t-s) \vec{f}(x, s-\rho) d s,
$$

где $\omega_{\rho}$ - усредняющее ядро С. Л. Соболева, $f(x, t)=0$ при $t<0$, и заметим, что $\left(\vec{v}_{\rho}, p_{\rho}\right)$ - решение задачи

$$
\begin{gathered}
\vec{v}_{\rho t}-\nu \Delta \vec{v}_{\rho}+\nabla p_{\rho}=\vec{f}_{\rho}, \quad \operatorname{div} \vec{v}_{\rho}=0, \\
\left.\vec{v}_{\rho}\right|_{t=0}=0,\left.\quad \vec{v}_{\rho}\right|_{x \in S}=0 .
\end{gathered}
$$

Ясно, что $\vec{v}_{\rho}, \vec{v}_{\rho t} \in C\left(0, T^{\prime} ; W_{2}^{2}(\Omega)\right), T^{\prime}<T$, а значит, $\vec{v}_{\rho t} \in C\left(0, T^{\prime} ; L_{q_{1}}(\Omega)\right)$, $1 / q_{1} \geqslant 1 / 2-2 / n$. Перенеся $\vec{v}_{\rho t}$ в правую часть уравнения и рассмотрев $\vec{v}_{\rho}$ как решение стационарной задачи Стокса с $\vec{f}_{\rho}-\vec{v}_{\rho t}$ в правой части, заключаем, что $\vec{v}_{\rho} \in C\left(0, T^{\prime} ; W_{q_{1}}^{2}(\Omega)\right)$. Затем можно показать, что $\vec{v}_{\rho} \in C\left(0, T^{\prime} ; W_{q_{2}}^{2}(\Omega)\right)$, $1 / q_{2} \geqslant 1 / q_{1}-2 / n \geqslant 1 / 2-4 / n$, и наконец, что $\vec{v}_{\rho} \in W_{q, r}^{2,1}\left(\Omega^{T}\right)$ с любыми $r>1, q \geqslant 2$. Переходя к пределу при $\rho \rightarrow 0$ и используя полученную выше априорную оценку, видим, что $\vec{v} \in W_{q, r}^{2,1}\left(\Omega^{T}\right), \nabla p \in L_{q, r}\left(Q^{T}\right)$. Чтобы получить тот же самый результат при $q<2$, нужно лишь оценить при $|x| \geqslant R \gg 1$ решение задачи Коши

$$
\begin{aligned}
\vec{u}_{t}-\nu \Delta \vec{u}+\nabla s= & \vec{f} \zeta_{0}+\vec{f}_{1}, \quad \operatorname{div} \vec{u}=\vec{v} \cdot \nabla \zeta_{0}, \\
\left.\vec{u}\right|_{t=0}=0, &
\end{aligned}
$$


где $\vec{u}=\zeta_{0} \vec{v}, s=\zeta_{0} p, \vec{f}_{1}=-2 \nu \nabla \vec{v} \nabla \zeta_{0}-\nu \vec{v}_{0} \nabla^{2} \zeta_{0}+s \nabla \zeta_{0}, \zeta_{0}-$ та же функция, что в $\S 1$. Мы имеем

$$
\vec{u}=\int_{0}^{t} \int_{\mathbb{R}^{n}} \mathbb{T}(x-y, t-\tau)\left(\vec{f} \zeta_{0}(y)+\vec{f}_{1}(y, \tau)\right) d y d \tau+\int_{\mathbb{R}^{n}} \nabla E(x-y)\left(\vec{v} \cdot \nabla \zeta_{0}(y)\right) d y .
$$

Отсюда следует конечность нормы

$$
\|\vec{u}\|_{L_{r}\left(0, T ; \dot{W}_{q}^{2}\left(B^{R}\right)\right)},
$$

где $B^{R}=\{|x|>R\}$, а так как $\int \vec{v} \cdot \nabla \zeta_{0} d y=0$, то

$$
\begin{aligned}
\int_{\mathbb{R}^{n-1}} \nabla E(x-y) \vec{v} \cdot \nabla \zeta_{0} d y \\
\quad=\int_{\mathbb{R}^{n-1}}\left(\nabla E(x-y)-\nabla E\left(x-y_{0}\right)\right) \vec{v} \cdot \nabla \zeta_{0} d y, \quad y_{0} \in \mathbb{R}^{n} \backslash \bar{\Omega}
\end{aligned}
$$

и можно заключить, что конечна и норма $\left\|\vec{v}_{t}\right\|_{L_{q, r}\left(B^{R} \times(0, T)\right)}$. Таким образом, $\vec{v} \in$ $W_{q, r}^{2,1}\left(Q^{T}\right), \nabla p \in L_{q, r}\left(Q^{T}\right)$, что и требовалось.

3.2. Задача для системы Стокса с ненулевой дивергенцией. Рассмотрим теперь более общую, чем (1.1), задачу

$$
\begin{gathered}
\vec{v}_{t}-\nu \Delta \vec{v}+\nabla p=\vec{f}, \quad \operatorname{div} \vec{v}=g, \quad x \in \Omega, \quad t \in[0, T), \\
\left.\vec{v}\right|_{t=0}=\vec{v}_{0}(x),\left.\quad \vec{v}\right|_{x \in S}=0 .
\end{gathered}
$$

ТЕОРема 3.1. Пусть $\Omega$ - ограниченная область, $g \in L_{r}\left(0, T ; W_{q}^{1}(\Omega)\right), \vec{f} \in$ $L_{q, r}\left(Q^{T}\right)$, существует производная $g_{t} \in L_{r}\left(0, T, W_{q}^{-1}(\Omega)\right), \vec{v}_{0} \in B_{q, r}^{2-2 / r}(\Omega)$ согласовано с нулем на граниче и выполняются условия согласования

$$
\begin{gathered}
\operatorname{div} \vec{v}_{0}(x)=g(x, 0), \\
\int_{\Omega} g(x, t) d x=0 .
\end{gathered}
$$

Тогда задача (3.13) имеет единственное решение $\vec{v} \in W_{q, r}^{2,1}\left(Q_{T}\right), \nabla p \in L_{q, r}\left(Q_{T}\right)$, и выполняется неравенство

$$
\begin{aligned}
& \|\vec{v}\|_{W_{q, r}^{2,1}\left(Q^{T}\right)}+\|\nabla p\|_{L_{q, r}\left(Q^{T}\right)} \\
& \quad \leqslant c\left(\|\vec{f}\|_{L_{q, r}\left(Q^{T}\right)}+\|g\|_{L_{r}\left(0, T ; W_{q}^{1}(\Omega)\right)}+\left\|g_{t}\right\|_{L_{r}\left(0, T ; W_{q}^{-1}(\Omega)\right)}+\left\|\vec{v}_{0}\right\|_{q, \Omega}^{2-2 / r}\right) .
\end{aligned}
$$


Tеорема 3.2. Предположим, что $\Omega$ - внешняя область, $\vec{f} \in L_{q, r}\left(Q^{T}\right), g \in$ $L_{r}\left(0, T ; \widetilde{W}_{q}^{1}(\Omega)\right)$, существует производная $g_{t} \in L_{r}\left(0, T ; \widetilde{W}_{q}^{-1}(\Omega)\right), \vec{v}_{0} \in \widetilde{B}_{q, r}^{2-2 / r}(\Omega)$ и выполнено условие согласования

$$
\operatorname{div} \vec{v}_{0}(x)=g(x, 0) .
$$

Тогда задача (3.13) имеет единственное решение $\vec{v} \in \widetilde{W}_{q, r}^{2,1}\left(Q_{T}\right), \nabla p \in L_{q, r}\left(Q^{T}\right)$ $u$

$$
\begin{aligned}
& \|\vec{v}\|_{\widetilde{W}_{q, r}^{2,1}\left(Q^{T}\right)}+\|\nabla p\|_{L_{q, r}\left(Q^{T}\right)} \\
& \quad \leqslant c\left(\|\vec{f}\|_{L_{q, r}\left(Q^{T}\right)}+\|g\|_{L_{r}\left(0, T ; \widetilde{W}_{q}^{1}(\Omega)\right)}+\left\|g_{t}\right\|_{L_{r}\left(0, T ; \widetilde{W}_{q}^{-1}(\Omega)\right)}+\left\|\vec{v}_{0}\right\|_{\widetilde{B}_{q, r}^{2-2 / r}(\Omega)}\right) .
\end{aligned}
$$

Eсли $n \geqslant 3, q<n / 2$, то постоянная с не зависит от $T$.

Условия (3.14), (3.16) понимаются в обобщенном смысле как

$$
\lim _{t \rightarrow 0} \int_{\Omega}(\operatorname{div} \vec{V}(x, t)-g(x, t)) \varphi(x) d x=0
$$

для любого $\vec{V} \in W_{q, r}^{2,1}\left(Q^{T}\right)\left(\vec{V} \in \widetilde{W}_{q, r}^{2,1}\left(Q^{T}\right)\right.$ в случае внешней области) такого, что $\left.\vec{V}\right|_{t=0}=\vec{v}_{0}(x)$, и для любой гладкой финитной $\varphi(x)$. В частности, если $g(x, t)=0$, то $(3.18)$ влечет за собой

$$
0=\lim _{t \rightarrow 0} \int_{\Omega} \vec{V}(x, t) \cdot \nabla \varphi(x) d x=\int_{\Omega} \vec{v}_{0}(x) \cdot \nabla \varphi(x) d x
$$

что означает, что $\vec{v}_{0}$ удовлетворяет (возможно, в слабом смысле) условиям (1.2).

Как обычно,

$$
\|g\|_{L_{r}\left(0, T ; W_{q}^{-1}(\Omega)\right)}=\left(\int_{0}^{T}\|g(\cdot, t)\|_{W_{q}^{-1}(\Omega)}^{r} d t\right)^{1 / r}
$$

где

$$
\|g\|_{W_{q}^{-1}(\Omega)}=\sup _{\|\varphi\|_{W_{q^{\prime}}^{\prime}(\Omega)}=1}\left|\int_{\Omega} g \varphi d x\right|, \quad 1 / q^{\prime}=1-1 / q .
$$

Так же определяется норма $\|g\|_{L_{r}\left(0, T ; \widetilde{W}_{q}^{-1}(\Omega)\right)}$.

Теоремы 3.1 и 3.2 доказываются путем сведения задачи (3.13) к задаче (3.1) по схеме, которая проведена в работе [15] в случае ограниченной области $\Omega$. В случае внешней области нам потребуются следуюшие вспомогательные предложения. 
ПРЕДЛОЖЕНИЕ 3.1. Пусть $\Omega$ - внешняя область. Для любой функиии $и \in$ $\widetilde{W}_{q}^{1}(\Omega)$ справедливы неравенства:

$$
\|u\|_{L_{s}(\Omega)} \leqslant c\|u\|_{\widetilde{W}_{q}^{1}(\Omega)},
$$

əде $1 / s=1 / q-1 / n, q<n$;

$$
\left(\int_{\Omega}|u(x)|^{q} \frac{d x}{\left|x-x_{0}\right|^{q}}\right)^{1 / q} \leqslant c\|u\|_{\widetilde{W}_{q}^{1}(\Omega)},
$$

əде $x_{0} \in \mathbb{R}^{n} \backslash \bar{\Omega}, q>n ; u$

$$
\left(\int_{\Omega}|u(x)|^{n} \frac{d x}{\left|x-x_{0}\right|^{n}\left(\log \frac{\left|x-x_{0}\right|}{h}\right)^{n}}\right)^{1 / n} \leqslant c\|u\|_{\widetilde{W}_{n}^{1}(\Omega)},
$$

əде $h<\operatorname{dist}\left(x_{0}, S\right)$.

ПРЕДЛОЖЕНИЕ 3.2. Всякая функция $u \in \widetilde{W}_{q}^{1}(\Omega)$ может быть аппрожсимирована в норме $\widetilde{W}_{q}^{1}(\Omega)$ финитными функциями $u(x) \psi_{m}(x)$, где

$$
\begin{array}{ll}
\psi_{m}(x)=\zeta\left(\frac{|x|}{m}\right), & \text { если } q \neq n \\
\psi_{m}(x)=\zeta\left(\log \left(\frac{\log \left|x-x_{0}\right| / h}{m}\right)\right), & \text { ecлu } q=n
\end{array}
$$

$\zeta(t)$ - функиия из $C^{\infty}\left(\mathbb{R}_{+}\right)$с носителем в интервале $0 \leqslant t<1$, обладающая свойствами: $\zeta(t)=1$ для $0 \leqslant t \leqslant 1 / 2,0 \leqslant \zeta(t) \leqslant 1$.

Неравенство (3.19) - это хорошо известная теорема вложения Соболева, а (3.20) и (3.21) - неравенства Харди, так что доказательство предложения 3.1 не приводится. Предложение 3.2 следует из предложения 3.1 .

ПРЕДЛОЖЕНИЕ 3.3. Пусть $\Omega$ - внешняя область. Для всякой функиии $\vec{v}_{0} \in$ $\widetilde{B}_{q, r}^{2-2 / r}(\Omega)$ существует функиия $\vec{V}_{0} \in \widetilde{W}_{q, r}^{2,1}\left(Q^{T}\right)$ такая, что $\vec{V}_{0}(x, 0)=\vec{v}_{0}(x)$, $\left.\vec{V}_{0}(x, t)\right|_{x \in S}=0 u$

$$
\left\|\vec{V}_{0}\right\|_{\widetilde{W}_{q, r}^{2,1}\left(Q^{T}\right)} \leqslant c\left\|\vec{v}_{0}\right\|_{\widetilde{B}_{q, r}^{2-2 / r}(\Omega)} .
$$

Если $n \geqslant 3, q<n / 2$, то постоянная с от $T$ не зависит.

ДокАЗАТЕЛЬСТво. Пусть $\zeta_{0}, \zeta_{1}, \ldots, \zeta_{M}-$ разбиение единицы из $\S 1$. Для каждого $k=0, \ldots, M$ определим функцию $\vec{V}_{k}$ по формулам

$$
\begin{aligned}
& \vec{V}_{0}(x, t)=\int_{\mathbb{R}^{n}} \Gamma(x-\xi, t) \vec{v}_{0} \zeta_{0}(\xi) d \xi \\
& \vec{V}_{k}(z, t)=\int_{\mathbb{R}^{n}} \Gamma(z-\xi, t) \vec{v}_{k}^{(-)}(\xi) d \xi, \quad k=1, \ldots, M,
\end{aligned}
$$


где $\vec{v}_{k}^{(-)}$- нечетное продолжение $\vec{v}_{k}^{\prime}(\xi)=\vec{v}_{0}\left(T_{k}^{-1} \xi\right) \zeta_{k}\left(T_{k}^{-1} \xi\right)$ на все $\mathbb{R}^{n}$. Вследствие неравенства $(2.22)$

$$
\begin{aligned}
& \left\|\vec{V}_{0}\right\|_{\dot{W}_{q, r}^{2,1}\left(\Pi^{T}\right)} \leqslant c\left\|\vec{v}_{0} \zeta_{0}\right\|_{\dot{B}_{q, r}^{2-2 / r}\left(\mathbb{R}^{n}\right)}, \\
& \left\|\vec{V}_{k}\right\|_{\dot{W}_{q, r}^{2,1}\left(\Pi^{T}\right)} \leqslant c\left\|\vec{v}_{k}^{(-)}\right\|_{\dot{B}_{q, r}^{2-2 / r}\left(\mathbb{R}^{n}\right)}, \quad k=1, \ldots, M,
\end{aligned}
$$

кроме того, для любой ограниченной подобласти $\Omega_{\mathbb{R}^{n}}^{\prime}$

$$
\begin{aligned}
\left\|\vec{V}_{0}\right\|_{L_{q, r}\left(\Omega^{\prime} \times\left(0, t_{0}\right)\right)} & \leqslant\left\|\vec{v}_{0} \zeta_{0}\right\|_{L_{q}\left(\Omega^{\prime}\right)}+t_{0}\left\|\vec{V}_{0 t}\right\|_{L_{q, r}\left(\Omega^{\prime} \times\left(0, t_{0}\right)\right)} \\
& \leqslant\left\|\vec{v}_{0} \zeta_{0}\right\|_{L_{q}\left(\Omega^{\prime}\right)}+c t_{0}\left\|\vec{v}_{0} \zeta_{0}\right\|_{\dot{B}_{q, r}^{2-2 / r}\left(\mathbb{R}^{n}\right)}, \\
\left\|\vec{V}_{k}\right\|_{L_{q, r}\left(\Omega^{\prime} \times\left(0, t_{0}\right)\right)} & \leqslant\left\|\vec{v}_{k}^{(-)}\right\|_{L_{q}\left(\Omega^{\prime}\right)}+c t\left\|\vec{v}_{k}^{(-)}\right\|_{\dot{B}_{q, r}^{2-2 / r}\left(\mathbb{R}^{n}\right)},
\end{aligned}
$$

а если $n \geqslant 3, q<n / 2$, то вследствие теоремы вложения С. Л. Соболева

$$
\begin{gathered}
\left\|\vec{V}_{j}\right\|_{L_{q, r}\left(\Omega^{\prime} \times\left(0, t_{0}\right)\right)} \leqslant c\left\|\vec{V}_{j}\right\|_{L_{s, r}\left(\Pi^{T}\right)} \leqslant c\left\|\vec{V}_{j}\right\|_{\dot{W}_{q, r}^{2,1}\left(\Pi^{T}\right)}, \\
j=0,1, \ldots, M, \quad 1 / s=1 / q-2 / n
\end{gathered}
$$

с постоянными, не зависяшими от $T$. Функцию $\vec{V}$ определим равенством

$$
\vec{V}(x, t)=\chi_{0} \vec{V}_{0}(x, t)+\sum_{j=1}^{M} \chi_{j}(x) \vec{V}_{k}\left(T_{k}^{-1} x, t\right),
$$

где $\chi_{k}(x)$ - гладкие функции с компактньми носителями такие, что $\chi_{k}(x) \zeta_{k}(x)=$ $\zeta_{k}(x)$ и $\operatorname{supp} \chi_{0} \subset \Omega$. Ясно, что $\vec{V}$ обладает всеми необходимыми свойствами. Предложение доказано.

Приступим теперь к доказательству теорем 3.1 и 3.2 . Будем искать решение задачи (3.13) в виде суммы

$$
\begin{aligned}
& \vec{v}=\vec{v}_{1}+\vec{v}_{2}+\vec{v}_{3}, \\
& p=p_{2}+p_{3},
\end{aligned}
$$

где $\vec{v}_{1}=\vec{V}_{0}$ - векторное поле, определенное в предложении 3.3 , а $\left(\vec{v}_{2}, p_{2}\right)$ - решение стационарной задачи

$$
\begin{gathered}
-\nu \Delta \vec{v}_{2}+\nabla p_{2}=0, \quad \operatorname{div} \vec{v}_{2}=g-\operatorname{div} \vec{v}_{1} \equiv g^{\prime}, \quad x \in \Omega, \\
\left.\vec{v}\right|_{S}=0 .
\end{gathered}
$$

Тогда для $\left(\vec{v}_{3}, p_{3}\right)$ получаем задачу типа $(3.1)$ :

$$
\begin{gathered}
\vec{v}_{3 t}-\nu \Delta \vec{v}_{3}+\nabla p_{3}=\vec{f}-\vec{v}_{1 t}+\nu \Delta \vec{v}_{1}-\vec{v}_{2 t} \equiv \vec{f}, \\
\operatorname{div} \vec{v}_{3}=0, \quad x \in \Omega, \quad t \in(0, T), \\
\left.\vec{v}_{3}\right|_{t=0}=0,\left.\quad \vec{v}_{3}\right|_{x \in S}=0 .
\end{gathered}
$$


Функция $\vec{v}_{1}$ была оценена в предложении 3.3. Рассмотрим задачу (3.24). Аппроксимируем $g^{\prime}$ финитными функциями $g_{m}^{\prime}=g^{\prime}(x) \psi_{m}(x)$, как указано в предложении 3.2 , и определим $\left(\vec{v}_{2}^{(m)}, p_{2}^{(m)}\right)$ как решение задачи

$$
\begin{gathered}
-\nu \Delta \vec{v}_{2}^{(m)}+\nabla p_{2}^{(m)}=0, \quad \operatorname{div} \vec{v}_{2}^{(m)}=g_{m}^{\prime}(x, t), \quad x \in \Omega, \\
\left.\vec{v}_{2}^{(m)}\right|_{S}=0, \quad \vec{v}_{2}^{(m)}, p_{2}^{(m)} \underset{|x| \rightarrow \infty}{\longrightarrow} 0 \quad\left(\vec{v}_{2}^{(m)} \underset{|x| \rightarrow \infty}{\longrightarrow} \text { const при } n=2\right) .
\end{gathered}
$$

Оно может быть найдено в форме

$$
\begin{aligned}
\vec{v}_{2}^{(m)}(x, t) & =\int_{\Omega} \nabla E(x-y) g_{m}^{\prime}(y, t) d y+\vec{w}_{2}^{(m)}(x, t) \\
& =\int_{\Omega} E(x-y) \nabla g_{m}^{\prime}(y, t) d y+\int_{\Omega} E(x-y) \vec{n}(y) g^{\prime}(y, t) d S+\vec{w}_{2}^{(m)}(x, t), \\
& p_{2}^{(m)}(x, t)=\nu g_{m}^{\prime}(x, t)+r_{2}^{(m)}(x, t)
\end{aligned}
$$

где $\vec{w}_{2}^{(m)}, r_{2}^{(m)}$ - решение задачи с неоднородньм краевьм условием

$$
\begin{gathered}
-\nu \Delta \vec{w}_{2}^{(m)}+\nabla r_{2}^{(m)}=0, \quad \operatorname{div} \vec{w}_{2}^{(m)}=0, \\
\left.\vec{w}_{2}^{(m)}\right|_{x \in S}=-\left.\vec{w}_{1}^{(m)}\right|_{x \in S}, \\
\vec{w}_{2}^{(m)}(x, t) \underset{|x| \rightarrow \infty}{\longrightarrow} 0(n \geqslant 3), \quad \vec{w}_{2}^{(m)}(x, t) \underset{|x| \rightarrow \infty}{\longrightarrow} \operatorname{const}(n=2) .
\end{gathered}
$$

При $n \geqslant 3, q<n / 2$ имеет место неравенство

$$
\left\|\vec{v}_{2}^{(m)}(\cdot, t)\right\|_{\widetilde{W}_{q}^{2}(\Omega)}+\left\|\nabla p_{2}^{(m)}\right\|_{L_{q}(\Omega)} \leqslant c\left\|g_{m}^{\prime}\right\|_{\widetilde{W}_{q}^{1}(\Omega)} \leqslant c\left\|g^{\prime}\right\|_{\widetilde{W}_{q}^{1}(\Omega)}
$$

(ср. с [25; теорема 1$])$, а значит,

$$
\left\|\vec{v}_{2}^{(m)}\right\|_{\widetilde{W}_{q, r}^{2,1}\left(Q^{T}\right)}+\left\|\nabla p_{2}^{(m)}\right\|_{L_{q, r}\left(Q^{T}\right)} \leqslant c\left\|g_{m}^{\prime}\right\|_{L_{r}\left(0, T ; \widetilde{W}_{q}^{1}(\Omega)\right)}
$$

с постоянной, не зависяшей от $T$.

При $q \geqslant n / 2$ эта оценка не имеет места, но $\vec{v}_{2}^{(m)} \in \widetilde{W}_{q, r}^{2,1}\left(Q^{T}\right), \nabla p^{(m)} \in L_{q, r}\left(Q^{T}\right)$ при всех $m \geqslant 1$.

Рассмотрим вспомогательную задачу

$$
\begin{gathered}
-\nu \Delta \vec{\varphi}(x)+\nabla \pi(x)=\vec{f}(x), \quad \operatorname{div} \vec{\varphi}(x)=0, \quad x \in \Omega, \\
\left.\vec{\varphi}\right|_{S}=0, \quad \vec{\varphi}(x), \pi(x) \underset{|x| \rightarrow \infty}{\longrightarrow} 0(n \geqslant 3), \\
\vec{\varphi}(x) \rightarrow \text { const }(n=2)
\end{gathered}
$$

для некоторого гладкого финитного векторного поля $\vec{f}(x)$. Интегрируя по частям, получаем

$$
\begin{aligned}
\int_{\Omega} \vec{v}_{2}^{(m)}(x, t) \cdot \vec{f}(x) d x & =-\int_{\Omega} g_{m}^{\prime}(x, t) \pi(x) d x+\int_{\Omega} \vec{\varphi}(x) \cdot\left(-\nu \Delta \vec{v}_{2}^{(m)}+\nabla p_{2}^{(m)}\right) d x \\
& =-\int_{\Omega} g_{m}^{\prime}(x, t) \pi(x) d x
\end{aligned}
$$


Вследствие (3.18) интеграл в правой части стремится к нулю при $t \rightarrow 0$, кроме того, он имеет производную по $t$ из $L_{r}(0, T)$. При почти всех $t \in(0, T)$ имеем

$$
\begin{aligned}
\left|\frac{d}{d t} \int_{\Omega} g_{m}^{\prime} \pi(x) d x\right| & \leqslant c\left\|g_{t}^{\prime}(\cdot, t)\right\|_{\widetilde{W}_{q}^{-1}(\Omega)}\left\|\psi_{m} \pi\right\|_{\widetilde{W}_{q^{\prime}}^{1}(\Omega)} \\
& \leqslant\left(\left\|g_{t}(\cdot, t)\right\|_{\widetilde{W}_{q}^{-1}(\Omega)}+\left\|\vec{v}_{1 t}(\cdot, t)\right\|_{L_{q}(\Omega)}\right)\|\pi\|_{\widetilde{W}_{q^{\prime}}^{1}(\Omega)} .
\end{aligned}
$$

В $[25]$ показано, что при любом $q^{\prime}>1$ в пространстве $L_{q^{\prime}}(\Omega)$ сушествуют такие плотные множества финитных векторных полей $\vec{f}$, что решение задачи $(3.26)$ подчиняется неравенству

$$
\|\vec{\varphi}\|_{\widetilde{W}_{q^{\prime}}^{2}(\Omega)}+\|\pi\|_{\widetilde{W}_{q^{\prime}}^{1}\left(\Omega_{1}\right)} \leqslant c\|\vec{f}\|_{L_{q^{\prime}}(\Omega)} ;
$$

следовательно, для любого $\vec{f}$ из соответствующего плотного множества имеем

$$
\left|\frac{d}{d t} \int_{\Omega} \vec{v}_{2}^{(m)}(x, t) \cdot \vec{f}(x) d x\right| \leqslant\left(\left\|g_{t}(\cdot, t)\right\|_{\widetilde{W}_{q}^{-1}(\Omega)}+\left\|\vec{v}_{1 t}(\cdot, t)\right\|_{L_{q}(\Omega)}\right)\|\vec{f}\|_{L_{q^{\prime}}(\Omega)} .
$$

Это значит, что $\vec{v}_{2_{t}}^{(m)} \in L_{q, r}\left(Q^{T}\right)$ и

$$
\left\|\vec{v}_{2 t}^{(m)}\right\|_{L_{q, r}(\Omega)} \leqslant c\left(\left\|g_{t}\right\|_{L_{r}\left(0, T ; \widetilde{W}_{q}^{-1}(\Omega)\right)}+\left\|\vec{v}_{1 t}\right\|_{L_{q, r}(\Omega)}\right) .
$$

Так как $\vec{v}_{2}^{(m)}(x, 0)=0$, то

$$
\left\|\vec{v}_{2}^{(m)}(\cdot, t)\right\|_{L_{q}(\Omega)} \leqslant \int_{0}^{t}\left\|\vec{v}_{2 t}^{(m)}(\cdot, \tau)\right\|_{L_{q}(\Omega)} d \tau,
$$

а значит,

$$
\left\|\vec{v}_{2}^{(m)}(\cdot, t)\right\|_{L_{q, r}(\Omega)} \leqslant c T\left(\left\|g_{t}\right\|_{L_{r}\left(0, T ; \widetilde{W}_{q}^{-1}(\Omega)\right)}+\left\|\vec{v}_{1 t}\right\|_{L_{q, r}(\Omega)}\right) .
$$

Воспользовавшись теперь справедливой при всех $q>1$ оценкой

$$
\left\|\vec{v}_{2}^{(m)}(\cdot, t)\right\|_{\dot{W}_{q}^{2}(\Omega)}+\left\|\nabla p_{2}^{(m)}\right\|_{L_{q}(\Omega)} \leqslant c\left(\left\|g_{m}^{\prime}\right\|_{\dot{W}_{q}^{1}(\Omega)}+\left\|\vec{v}_{2}^{(m)}(\cdot, t)\right\|_{L_{q}(\Omega)}\right)
$$

и переходя к пределу при $m \rightarrow \infty$, заключаем, что задача (3.24) имеет решение $\vec{v}_{2} \in$ $\widetilde{W}_{q, r}^{2,1}\left(Q^{T}\right), \nabla p_{2} \in L_{q, r}\left(Q^{T}\right)$, для которого справедливо неравенство

$$
\begin{aligned}
& \left\|\vec{v}_{2}\right\|_{\widetilde{W}_{q, r}^{2,1}\left(Q^{T}\right)}+\left\|\nabla p_{2}\right\|_{L_{q, r}\left(Q^{T}\right)} \\
& \quad \leqslant c\left(\|g\|_{L_{r}\left(0, T ; \widetilde{W}_{q}^{1}(\Omega)\right)}+\left\|g_{t}\right\|_{L_{r}\left(0, T ; \widetilde{W}_{q}^{-1}(\Omega)\right)}+\left\|\vec{v}_{1}\right\|_{\widetilde{W}_{q, r}^{2,1}\left(Q^{T}\right)}\right) \\
& \quad \leqslant c\left(\|g\|_{L_{r}\left(0, T ; \widetilde{W}_{q}^{1}(\Omega)\right)}+\left\|g_{t}\right\|_{L_{r}\left(0, T ; \widetilde{W}_{q}^{-1}(\Omega)\right)}+\left\|\vec{v}_{0}\right\|_{\widetilde{B}_{q, r}^{2-2 / r}(\Omega)}\right)
\end{aligned}
$$

причем постоянная $c$ не зависит от $T$, если $n \geqslant 3$ и $q<n / 2$ (благодаря (3.23) и (3.25)). Таким образом, задача (3.13) свелась к (3.1). Оценка (3.17) следует из (3.22), (3.28), (1.10) (c $\left.\vec{v}_{0}=0\right)$. Теорема 3.2 доказана. 


\section{§ 4. Оценка резольвенты оператора Стокса}

Переходим к рассмотрению задачи (1.3). Приведем оценку решения этой задачи, полученную в работе [4].

ПРЕДЛОЖЕНИЕ 4.1. Для любого решения $\vec{u} \in W_{q}^{2}(\Omega)$ задачи (1.3) справедлива оченка (1.11), если $\lambda \in \Sigma_{\rho, \omega}=\{|\arg \lambda| \leqslant \pi / 2+\omega,|\lambda|>\rho\}$ при некоторых $\rho>0 u$ $\omega \in(0, \pi / 2)$.

ДокАЗАТЕльство. Пусть $\eta(\sigma)$ - гладкая монотонная функция положительного аргумента, равная нулю при $\sigma \leqslant 1 / 2$ и единице при $\sigma \geqslant 1$. Если $\vec{u}(x), s(x)$ - решение задачи (1.3), то

$$
\vec{v}(x, t)=\vec{u}(x) e^{\lambda t}(1-\eta(t h)), \quad p(x, t)=s(x) e^{\lambda t}(1-\eta(t h)),
$$

$h>0$ - решение задачи (3.1) при

$$
\vec{f}(x, t)=\vec{f}(x) e^{\lambda t}(1-\eta(t h))-h \eta^{\prime}(t h) \vec{u}(x) e^{-\lambda t}
$$

и оценка (3.12) с $r=q$ влечет за собой

$$
\begin{aligned}
& |\lambda|\|\vec{u}\|_{L_{q}(\Omega)}+\|\vec{u}\|_{\dot{W}_{q}^{2}(\Omega)}+\|\nabla s\|_{L_{q}(\Omega)} \\
& \quad \leqslant c_{1}\|\vec{f}\|_{L_{q}(\Omega)}+\left(c_{2}+h \psi^{1 / q}(\lambda, h, T) \Phi^{-1 / q}(\lambda, h, T)\left(c_{1}+1\right)\right)\|\vec{u}\|_{L_{q}(\Omega)},
\end{aligned}
$$

где

$$
\begin{aligned}
& \Phi(\lambda, h, T)=\int_{0}^{T}(1-\eta(t h))^{q} e^{q t \operatorname{Re} \lambda} d t \\
& \Psi(\lambda, h, T)=\int_{0}^{T}\left(\eta^{\prime}(t h)\right)^{q} e^{q t \operatorname{Re} \lambda} d t
\end{aligned}
$$

Потребуем, чтобы $\rho>c_{2}$, и подберем параметры $h$ и $T$ так, чтобы

$$
|\lambda|^{-1} h \Psi^{1 / q} \Phi^{-1 / q}\left(c_{1}+1\right) \leqslant \beta, \quad \beta<1-c_{2} / \rho,
$$

тогда

$$
c_{2}+h\left(c_{1}+1\right) \Psi^{1 / q} \Phi^{-1 / q} \leqslant c_{2}+\beta|\lambda| \leqslant \gamma|\lambda|
$$

с $\gamma=\beta+c_{2} / \rho<1$, а значит, из (4.1) будет следовать

$$
(1-\gamma)|\lambda|\|\vec{u}\|_{L_{q}(\Omega)}+\|\vec{u}\|_{\dot{W}_{q}^{2}(\Omega)}+\|\nabla s\|_{L_{q}(\Omega)} \leqslant c_{1}\|\vec{f}\|_{L_{q}(\Omega)},
$$

откуда вытекает (1.11).

Заметим, что $\Phi$ и $\Psi$ удовлетворяют неравенствам

$$
\begin{gathered}
\Phi \geqslant \int_{1 / h}^{T} e^{q t \operatorname{Re} \lambda} d t=\frac{e^{q T \operatorname{Re} \lambda}-e^{q \operatorname{Re} \lambda / h}}{q \operatorname{Re} \lambda}, \\
\Psi \leqslant \max \left|\eta^{\prime}(s)\right| \int_{1 / 2 h}^{1 / h} e^{q t \operatorname{Re} \lambda} d t \leqslant \max \left|\eta^{\prime}(s)\right|^{q} \frac{e^{q \operatorname{Re} \lambda / h}-e^{q \operatorname{Re} \lambda / 2 h}}{q \operatorname{Re} \lambda},
\end{gathered}
$$


если $\operatorname{Re} \lambda \neq 0$, и

$$
\Phi \geqslant T-\frac{1}{h}, \quad \Psi \leqslant \frac{2}{h} \max \left|\eta^{\prime}(s)\right|^{q},
$$

если $\operatorname{Re} \lambda=0$. Поэтому при $\operatorname{Re} \lambda \geqslant 0$ левая часть в (4.2) может быть сделана сколь угодно малой, если положить $h=|\operatorname{Re} \lambda|$ при $\operatorname{Re} \lambda>0, h=1$ при $\operatorname{Re} \lambda=0$ и выбрать $T$ достаточно большим: $T \operatorname{Re} \lambda \gg 1$ при $\operatorname{Re} \lambda>0, T \gg 1$ при $\operatorname{Re} \lambda=0$; в случае $\operatorname{Re} \lambda<0$ можно взять $h=|\operatorname{Re} \lambda|, T|\operatorname{Re} \lambda|>2$ и потребовать, чтобы отношение $h /|\lambda|=$ $|\operatorname{Re} \lambda| /|\lambda|$ было достаточно малым, что имеет место при малом $\omega$. Во всех случаях неравенство (4.2) может быть удовлетворено. Предложение доказано.

Из доказательства ясно, что если неравенство (3.12) справедливо с $c_{2}=0$ и $c_{1}$ не зависит от $T$, то можно взять выше $\rho=0$ (см. [11; теорема 4.3]).

Для ограниченной области справедлива и более сильная, чем (1.11), оценка решений задачи (1.3), а именно,

$$
(|\lambda|+1)\|\vec{u}\|_{L_{q}(\Omega)}+\sum_{|j|=2}\left\|D^{j} \vec{u}\right\|_{L_{q}(\Omega)}+\|\nabla s\|_{L_{q}(\Omega)} \leqslant c\|\vec{f}\|_{L_{q}(\Omega)}
$$

причем $\lambda$ может принадлежать множеству

$$
\Sigma_{\omega, 0} \cup\{-b<\operatorname{Re} \lambda \leqslant 0\}=\Sigma_{\omega, 0, b},
$$

где $b$ - некоторое положительное число. Она вытекает из предложения 4.1 и из несложного дополнительного исследования задачи (1.3) с $|\lambda|<\rho$.

ПРЕДЛОЖЕНИЕ 4.2. Если $\Omega$ - ограниченная область, то решение задачи (1.3) $(\vec{u}, s)$ подчиняется неравенству (4.3) для всех $\lambda \in \Sigma_{\omega, 0, b}$.

ДокАЗАТЕЛЬСтво. Достаточно ограничиться рассмотрением случая $|\lambda|<\rho$, и тогда (4.3) сводится к оценке

$$
\|\vec{u}\|_{W_{q}^{2}(\Omega)}+\|\nabla s\|_{L_{q}(\Omega)} \leqslant c\|\vec{f}\|_{L_{q}(\Omega)}
$$

Оператор Стокса не имеет собственных значений при $-b<\operatorname{Re} \lambda<0$ с некоторым $b>0$, откуда следует разрешимость задачи (4.3) и оценка (4.4) при $q=2$, когда $\lambda$ удовлетворяет всем указанным выше ограничениям. По теореме о гладкости решений эллиптических краевых задач (4.4) имеет место и при $q>2$. При $q<2$ следует воспользоваться соображениями двойственности. Если $\vec{f}$ принадлежит плотному в $L_{q}(\Omega)$ множеству гладких векторных полей, то задача (1.3) имеет решение $\vec{u} \in W_{2}^{2}(\Omega), \nabla s \in L_{2}(\Omega)$. Возьмем произвольное $\vec{g} \in L_{q^{\prime}}(\Omega)$; так как $q^{\prime}>2$, то задача

$$
\lambda \vec{\varphi}-\nu \Delta \vec{\varphi}+\nabla \pi=\vec{g}, \quad \operatorname{div} \vec{\varphi}=0,\left.\quad \vec{\varphi}\right|_{S}=0
$$

имеет единственное решение $\vec{\varphi} \in W_{q^{\prime}}^{2}(\Omega), \nabla \pi \in L_{q^{\prime}}(\Omega)$, и справедливо равенство

$$
\int_{\Omega} \vec{u} \cdot \vec{g} d x=\int_{\Omega}(\lambda \vec{\varphi}-\nu \Delta \vec{\varphi}) \cdot \vec{u} d x=\int_{\Omega}(\lambda \vec{u}-\nu \Delta \vec{u}) \cdot \vec{\varphi} d x=\int_{\Omega} \vec{f} \cdot \vec{\varphi} d x .
$$


Отсюда следует

$$
\left|\int_{\Omega} \vec{u} \cdot \vec{g} d x\right| \leqslant c\|\vec{f}\|_{L_{q}(\Omega)}\|\vec{\varphi}\|_{L_{q^{\prime}}(\Omega)} \leqslant c\|\vec{f}\|_{L_{q}(\Omega)}\|\vec{g}\|_{L_{q^{\prime}}(\Omega)},
$$

а значит, $\|\vec{u}\|_{L_{q}(\Omega)} \leqslant c\|\vec{f}\|_{L_{q}(\Omega)}$. Рассмотрев $\vec{u}, s$ как решение задачи Стокса

$$
-\nu \Delta \vec{u}+\nabla s=\vec{f}-\lambda \vec{u}, \quad \operatorname{div} \vec{u}=0,\left.\quad \vec{u}\right|_{S}=0,
$$

получаем (4.3) и при $q<2$, что и завершает доказательство предложения.

Переходим к краткому изложению основного результата работы [8]. В ней используются оценка (1.11) для ограниченной области, аналогичная оценка Мак-Крекен [19] для всего пространства $\mathbb{R}^{n}$ и следуюшее вспомогательное утверждение.

ПреДЛОЖЕНИЕ 4.3. Пусть $\Omega$ - внешняя область. При любом гладком финитном $\vec{f}$ и любом $\lambda \in \Sigma_{\omega, 0}, 0<|\lambda| \leqslant \rho$, задача (1.3) имеет решение $\vec{u} \in W_{q}^{2}(\Omega)$, $\nabla s \in L_{q}(\Omega)$ с любымм $q>1, u$

$$
\|\vec{u}\|_{W_{q}^{2}(\Omega)}+\|\nabla s\|_{L_{q}(\Omega)} \leqslant c\|\vec{f}\|_{L_{q}(\Omega)} .
$$

Доказательство этого предложения аналогично доказательству предложения 4.2; для оценки решения задачи Стокса во внешней области следует воспользоваться результатами работы [25].

Основной результат работы [8] состоит в следующем.

ПреДлоЖениЕ 4.4. Решение задачи (1.3) во внешней области $\Omega \subset \mathbb{R}^{n}, n \geqslant 3$, подчиняется неравенству (1.13) при любом $\lambda \in \Sigma_{\omega, 0} \quad \forall \omega>0$.

ДокАЗАТЕльСтво. Достаточно доказать (1.13) при дополнительном предположении $|\lambda| \leqslant \rho$. Кроме того, сначала предполагается, что $q<n / 2$, и в этом случае устанавливается равносильное неравенство (1.11). Рассуждение ведется от противного. Если (1.11) не имеет места, то существуют такие последовательности $\lambda_{m}, \vec{u}_{m}, s_{m}$, $m=1,2, \ldots$, что $\lambda_{m} \in \Sigma_{\omega, 0}, 0<\left|\lambda_{m}\right| \leqslant \rho$,

$$
\begin{gathered}
\vec{u}_{m} \in W_{q}^{2}(\Omega), \quad \nabla s_{m} \in L_{q}(\Omega), \\
\left\|\lambda_{m} \vec{u}_{m}\right\|_{L_{q}(\Omega)}+\left\|\vec{u}_{m}\right\|_{\dot{W}_{q}^{2}(\Omega)}+\left\|\nabla s_{m}\right\|_{L_{q}(\Omega)}=1, \quad m=1,2, \ldots, \\
\left\|\lambda_{m} \vec{u}_{m}-\nu \Delta \vec{u}_{m}+\nabla s_{m}\right\|_{L_{q}(\Omega)} \underset{m \rightarrow \infty}{\longrightarrow} 0 .
\end{gathered}
$$

Так как $q<n / 2$, то отсюда следует ограниченность суммы норм

$$
\left\|\vec{u}_{m}\right\|_{L_{r}(\Omega)}+\left\|\nabla \vec{u}_{m}\right\|_{L_{r_{1}}(\Omega)}+\left\|s_{m}\right\|_{L_{r_{1}}(\Omega)},
$$

где $r, r_{1}$ определяются соотношениями $\frac{1}{r_{1}}=\frac{1}{q}-\frac{1}{n}, \frac{1}{r}=\frac{1}{q}-\frac{2}{n}$. Заметим, что $\frac{1}{r^{\prime}}=\frac{2}{n}+\frac{1}{q^{\prime}}$, так что $r^{\prime}<\frac{n}{2}$. 
Очевидно, сушествует подпоследовательность $m_{k}, k \rightarrow \infty$, такая, что $\lim \lambda_{m_{k}}=$ $\lambda_{0} \in \bar{\Sigma}_{\omega, 0},\left|\lambda_{0}\right| \leqslant \rho$, а $\vec{u}_{m_{k}}, D^{j} \vec{u}_{m_{k}}, \nabla s_{m_{k}}$ сходятся слабо в соответствуюших нормах к $\vec{u} \in L_{r}(\Omega), D^{j} \vec{u},|j|=2, \nabla s \in L_{q}(\Omega)$. Будем считать, что уже исходные последовательности $\lambda_{m}, \vec{u}_{m}, D^{j} \vec{u}_{m}, \nabla s_{m}$ сходятся в указанном смысле.

При любых финитных $\vec{\varphi} \in W_{r^{\prime}}^{2}(\Omega), \pi \in W_{r^{\prime}}^{1}(\Omega)$ имеем

$$
\begin{aligned}
0 & =\lim _{m \rightarrow \infty} \int_{\Omega}\left(\lambda_{m} \vec{u}_{m}-\nu \Delta \vec{u}_{m}+\nabla s_{m}\right) \cdot \vec{\varphi} d x \\
& =\lim _{m \rightarrow \infty} \int_{\Omega} \vec{u}_{m} \cdot\left(\lambda_{m} \vec{\varphi}-\nu \Delta \vec{\varphi}+\nabla \pi\right) d x \\
& =\int_{\Omega} \vec{u} \cdot\left(\lambda_{0} \vec{\varphi}-\nu \Delta \vec{\varphi}+\nabla \pi\right) d x .
\end{aligned}
$$

Легко видеть, что функции $\lambda_{0} \vec{\varphi}-\nu \Delta \vec{\varphi}+\nabla \pi$ при всевозможных указанных $\vec{\varphi}, \pi$ плотны в $L_{r^{\prime}}(\Omega)$ (это следует из предложения 4.3 или, в случае $\lambda_{0}=0$, из результатов работы [25], поскольку $r^{\prime}<n / 2$ ). Поэтому (4.9) влечет за собой $\vec{u}=0$.

Пусть $B_{1}$ и $B_{2}$ - шары такие, что $\mathbb{R}^{n} \backslash \Omega \subset B_{1} \subset B_{2}, \varphi_{1}(x)$ - функция из $C_{0}^{\infty}\left(\mathbb{R}^{n}\right)$, $\varphi_{1}(x)=1$ в $B_{1}, \varphi_{1}(x)=0$ в $\mathbb{R}^{n} \backslash B_{2}, 0 \leqslant \varphi_{1}(x) \leqslant 1$ и $\varphi_{2}(x)=1-\varphi_{1}(x)$. Положим $\vec{u}_{m}^{(1)}=\vec{u}_{m} \varphi_{1}+\vec{w}_{m}, \vec{u}_{m}^{(2)}=\vec{u}_{m} \varphi_{2}-\vec{w}_{m}, s_{m}^{(1)}=s_{m} \varphi_{1}, s_{m}^{(2)}=s_{m} \varphi_{2}$, где $\vec{w}_{m}$-векторное поле, удовлетворяюшее соотношениям

$$
\begin{gathered}
\operatorname{div} \vec{w}_{m}=-\operatorname{div}\left(\vec{u}_{m} \varphi_{1}\right)=-\nabla \varphi_{1} \cdot \vec{u}_{m}, \quad x \in B_{2} \backslash B_{1}, \\
\left.\vec{w}_{m}\right|_{\partial B_{1} \cup \partial B_{2}}=0 .
\end{gathered}
$$

Можно считать, что $\nabla \vec{\varphi}_{1}=0$ вблизи $\partial B_{1} \cup \partial B_{2}$. Тогда согласно [26] существует векторное поле $\vec{w}_{m} \in W_{q}^{2}\left(B_{2} \backslash B_{1}\right)$ с компактным носителем в $B_{2} \backslash B_{1}$ такое, что

$$
\left\|\vec{w}_{m}\right\|_{W_{q}^{2}\left(B_{2} \backslash B_{1}\right)} \leqslant c\left\|\varphi_{1} \vec{u}_{m}^{\prime}\right\|_{W_{q}^{1}\left(B_{2} \backslash B_{1}\right)} .
$$

Ясно, что вследствие компактности вложения $W_{q}^{2}\left(B_{2} \backslash B_{1}\right)$ в $W_{q}^{1}\left(B_{2} \backslash B_{1}\right)$ нормы в правой части стремятся к нулю при $m \rightarrow \infty$. Мы положим $\vec{w}_{m}=0$ вне $B_{2} \backslash B_{1}$. Легко видеть, что $\vec{u}_{m}^{(1)}$ и $\vec{u}_{m}^{(2)}$ являются решениями задач

$$
\begin{gathered}
\lambda_{m} \vec{u}_{m}^{(1)}-\nu \Delta \vec{u}_{m}^{(1)}+\nabla s_{m}^{(1)}=\varphi_{1}\left(\lambda_{m} \vec{u}_{m}-\nu \Delta \vec{u}_{m}+\nabla s_{m}\right)+\vec{w}_{m}^{(1)}(x), \\
\operatorname{div} \vec{u}_{m}^{(1)}=0, \quad x \in B_{2} \cap \Omega \equiv \Omega^{(1)}, \\
\left.\vec{u}^{(1)}\right|_{\partial \Omega^{(1)}}=0, \\
\lambda_{m} \vec{u}_{m}^{(2)}-\nu \Delta \vec{u}_{m}^{(2)}+\nabla s_{m}^{(1)}=\varphi_{2}\left(\lambda_{m} \vec{u}_{m}-\nu \Delta \vec{u}_{m}+\nabla s_{m}\right)+\vec{w}_{m}^{(2)}(x), \\
\operatorname{div} \vec{u}_{m}^{(2)}=0, \quad x \in \mathbb{R}^{n} \equiv \Omega^{(2)},
\end{gathered}
$$

где $\vec{w}_{m}^{(i)}$ - функции с носителями в $B_{2} \backslash B_{1}$, обладаюшие тем свойством, что

$$
\left\|\vec{w}_{m}^{(1)}\right\|_{L_{q}\left(B_{2} \backslash B_{1}\right)}+\left\|\vec{w}_{m}^{(2)}\right\|_{L_{q}\left(B_{2} \backslash B_{1}\right)} \underset{m \rightarrow \infty}{\longrightarrow} 0 .
$$


Вследствие неравенства (4.3) и упомянутого вьше результата Мак-Крекен имеем

$$
\begin{aligned}
& \left|\lambda_{m}\right|\left\|\vec{u}_{m}^{(i)}\right\|_{L_{q}\left(\Omega^{(j)}\right)}+\sum_{|j|=2}\left\|D^{j} \vec{u}_{m}^{(i)}\right\|_{L_{q}\left(\Omega^{(j)}\right)}+\left\|\nabla s_{m}^{(i)}\right\|_{L_{q}\left(\Omega^{(j)}\right)} \\
& \quad \leqslant c_{1}\left(\left\|\varphi_{i}\left(\lambda_{m} \vec{u}_{m}-\nu \Delta \vec{u}_{m}+\nabla s_{m}\right)\right\|_{L_{q}\left(\Omega^{i}\right)}+\left\|\vec{w}_{m}^{(i)}\right\|_{L_{q}\left(B_{2} \backslash B_{1}\right)}\right),
\end{aligned}
$$

$i=1,2$. Складьвая эти две оценки и принимая во внимание (4.7), (4.11), приходим к противоречивому неравенству $1 \leqslant \varepsilon_{m} \underset{m \rightarrow \infty}{\longrightarrow} 0$. Таким образом, доказано, что при $q<n / 2, n \geqslant 3$

$$
\|u\|_{L_{q}(\Omega)} \leqslant c|\lambda|^{-1}\|f\|_{L_{q}(\Omega)}, \quad \lambda \in \Sigma_{\omega, 0}
$$

Кроме того, хорошо известно, что (4.12) справедливо при $q=2$, а значит, в случае $n=3$, и при $3 / 2 \leqslant q \leqslant 2$ (вследствие интерполящионной теоремы Рисса-Торина). Наконец, для $q>2$ (4.12) легко выводится из соображений дуальности. Предложение доказано.

\section{СПИСОК ЛИТЕРАТУРЫ}

[1] К. К. Головкин, О. А. Ладыженская. О решениях нестационарной краевой задачи для уравнений Навье-Стокса // Труды МИАН. 1960. Т. 59. С. 100-114.

[2] К.К. Головкин, В.А. Солонников. О первой краевой задаче для нестационарных уравнений Навье-Стокса // Докл. АН СССР. 1961. Т. 140. № 2. С. 267-290.

[3] В.А. Солонников. Оценки решений нестационарной линеаризованной системы уравнений Навье-Стокса // Труды МИАН. 1964. Т. 70. С. 213-317.

[4] В.А. Солонников. Оценки решений нестационарной системш Навье-Стокса // Зап. научн. семин. ЛОМИ. 1973. Т. 38. С. 153-231.

[5] Y. Giga. Analyticity of the semigroup generated by the Stokes operator in $L_{r}$ spaces // Math. Z. 1981. V. 178. № 3. P. 297-329.

[6] Y. Giga, H. Sohr. On the Stokes operator in exterior domains // J. Fac. Sci. Univ. Tokyo Sect. IA Math. 1989. V. 36. №1. P. 103-130.

[7] Y. Giga, H. Sohr. Abstract $L^{p}$-estimates for the Cauchy problem with applications to the Navier-Stokes equations in exterior domains // J. Funct. Anal. 1991. V. 102. № 1. P. 72-94.

[8] W. Borchers, H. Sohr. On the semigroup of the Stokes operator for exterior domains in $L^{q}$-spaces // Math. Z. 1987. V. 196. № 3. P. 415-425.

[9] W. Borchers, W. Varnhorn. On the boundedness of the Stokes semigroup in two-dimensional exterior domains // Math. Z. 1993. V. 213. № 2. P. 275-299.

[10] П. Маремонти, В. А. Солонников. Об оценках решений нестационарной задачи Стокса в анизотропных пространствах С. Л. Соболева со смешанной нормой // Зап. научн. семин. ПОМИ. 1994. Т. 293. С. 124-150.

[11] P. Maremonti, V. A. Solonnikov. On nonstationary Stokes problem in exterior domains // Ann. Scuola Norm. Sup. Pisa Cl. Sci. (4). 1997. V. 24. № 3. P. 395-449.

[12] В. А. Солонников. Коэрцитивные оценки в $L_{p}$ решений начально-краевой задачи для обобщенной системы уравнений Стокса в полупространстве // Проблемы матем. анализа. 2000. T. 20. C. 243-270.

[13] В. А. Солонников. Оценки в $L_{p}$ решений начально-краевой задачи для обобщенной системы уравнений Стокса в ограниченной области // Проблемы матем. анализа. 2000. Т. 21. C. 211-254.

[14] G. A. Seregin. Some estimates near the boundary for solutions of the nonstationary linearized Navier-Stokes equations // Зап. научн. семин. ПОМИ. 2000. Т. 271. C. 204-223.

[15] V. A. Solonnikov. Estimates of solutions of the Stokes equations in S. L. Sobolev spaces with a mixed norm // Зап. научн. семин. ПОМИ. 2002. Т. 288. С. 204-231. 
[16] Я. С. Бугров. Функционалњные пространства со смешанной нормой // Изв. АН СССР. Cер. матем. 1971. Т. 35. № 5. C. 1137-1158.

[17] H. Sohr. The Navier-Stokes Equations. An Elementary Functional Analytic Approach. Basel: Birkhäuser, 2001.

[18] R. Farwig, H. Sohr. The stationary and nonstationary Stokes system in exterior domains with nonzero divergence and nonzero boundary values // Math. Methods Appl. Sci. 1994. V. 17. №4. P. 269-291.

[19] M. McCracken. The resolvent problem for the Stokes equations on halfspace in $L_{p} / /$ SIAM J. Math. Anal. 1981. V. 12. № 2. P. 201-228.

[20] О.В.Бесов, В.П. Ильин, С. М. Никольский. Интегральные представления функций и теоремы вложения. М.: Наука, 1975.

[21] A. P. Calderón, A. Zygmund. On singular integrals // Amer. J. Math. 1956. V. 78. P. $289-309$

[22] В.А. Солонников. Оценки решения одной начально-краевой задачи для линейной нестационарной системы уравнений Навье-Стокса // Зап. научн. семин. ЛОМИ. 1976. Т. 59. C. $178-254$.

[23] О. А. Ладыженская. Математические вопросы динамики вязкой несжимаемой жидкости. М.: Наука, 1970.

[24] В. А. Солонников. О разрешимости второй начально-краевой задачи для линейной нестационарной системы уравнений Навье-Стокса // Зап. научн. семин. ЛОМИ. 1977. Т. 69. C. $200-218$.

[25] П. Маремонти, В. А. Солонников. Об оценке решений системы Стокса во внешних областях // Зап. научн. семин. ЛОМИ. 1990. Т. 180. С. 105-120.

[26] М. Е. Боговский. Решение некоторых задач векторного анализа, связанных с операторами div и grad // Труды семин. С. Л. Соболева. 1980. № 1. С. 5-40.

С.-Петербургское отделение

Поступила в редакцию

Математического института им. В. А. Стеклова РАН

15.02.2003 